TRANSACTIONS OF THE

AMERICAN MATHEMATICAL SOCIETY

Volume 352, Number 4, Pages 1759-1796

S 0002-9947(99)02558-1

Article electronically published on December 10, 1999

\title{
AN APPROACH TO SYMMETRIZATION VIA POLARIZATION
}

\author{
FRIEDEMANN BROCK AND ALEXANDER YU. SOLYNIN
}

\begin{abstract}
We prove that the Steiner symmetrization of a function can be approximated in $L^{p}\left(\mathbb{R}^{n}\right)$ by a sequence of very simple rearrangements which are called polarizations. This result is exploited to develop elementary proofs of many inequalities, including the isoperimetric inequality in Euclidean space. In this way we also obtain new symmetry results for solutions of some variational problems. Furthermore we compare the solutions of two boundary value problems, one of them having a "polarized" geometry and we show some pointwise inequalities between the solutions. This leads to new proofs of well-known functional inequalities which compare the solutions of two elliptic or parabolic problems, one of them having a "Steiner-symmetrized" geometry. The method also allows us to investigate the case of equality in the inequalities. Roughly speaking we prove that the equality sign is valid only if the original problem has the symmetrized geometry.
\end{abstract}

\section{INTRODUCTION}

An isoperimetric theorem portrays the maximum or the minimum, subject to possible side conditions, of a functional whose domain is a collection of sets or functions (the "data" of the problem) and which has a special physical significance. In many important cases the extremum value of the functional is attained if the data have a simpler - symmetrized - geometry, i.e. the inequality can be expressed in terms of rearrangements. (The simplest example is the classical isoperimetric inequality in Euclidean space which says that among all sets of prescribed given volume the ball has the smallest perimeter.) Since the times of Polya and Szegö [PS] rearrangement techniques have turned out to be very fruitful in proving isoperimetric theorems in analysis and function theory. The articles [Ta1, [ALT2], Du3]-[Du4, [Bae2] and also the monograph Ka1 provide a large number of references.

Most results in the literature deal with the $(k, n)$-Steiner symmetrizations (for definition see section 4) and have been proved via the "method of level sets" (see [Ta1]- Ta2], [ADLT], We1], We2] and also [Ka1]). To employ this method one begins by assuming elementary facts about symmetrizations like the Hardy-Littlewood inequality (see (3.9)) or the isoperimetric inequality in $\mathbb{R}^{n}$ and then proceeds via sometimes extensive analysis using tools such as the coarea formula. One can prove

Received by the editors May 15, 1996.

1991 Mathematics Subject Classification. Primary 28D05, 58G35, 35A30, 35B05, 35B50, 35J60, 35K55, 26D10.

Key words and phrases. Steiner symmetrization, rearrangement, polarization, integral inequality, boundary value problem, comparison theorem, maximum principle, uniqueness theorem.

Research supported by Volkswagen-Stiftung, RiP-program. 
in this way Dirichlet-type inequalities, and also inequalities which compare the solutions of two boundary value problems, one of them having a "Steiner symmetrized" geometry.

Alvino, Lions and Trombetti ALT2 have recently introduced a different method which is based on the Trotter product formula for semigroups. This enabled them to prove comparison theorems for Steiner symmetrization in a very elegant way.

Another approach to symmetrization can be based on polarization. This simple type of rearrangement was introduced for plane sets by Wolontis Wo in 1952 and for functions by Baernstein and Taylor [BT] in 1976. In 1985 and later Dubinin Du1-Du4 often used polarization to derive inequalities for capacities in space. Solynin [So1] applied polarization to show the monotonicity of some capacities under one type of continuous Steiner symmetrization.

It was observed in $[\mathrm{BT}]$ that the proof of some integral inequalities becomes almost trivial if one replaces the symmetrization by the polarization. Furthermore, by using this result and some compactness argument, Baernstein proved a general convolution-type inequality for the $(k, n)$-Steiner and cap symmetrizations in a nice recent paper [Bae2]. Then he reduced the Dirichlet-type inequalities and comparison theorems for symmetrizations to this single inequality.

The present paper is a further step in this direction. Here we would like to warn the reader that some of the results which we derive for symmetrizations already exist in the literature. However, we hope to justify this by presenting new proofs which seem to be simpler than any of the old proofs.

We prove that the $(k, n)$-Steiner symmetrization of a nonnegative function $(1 \leq$ $k \leq n$ ) can be approximated in $L^{p}\left(\mathbb{R}^{n}\right)$ by a sequence of polarizations. Also we show in the particular case of a characteristic function of an open or compact set that the convergence can even be managed in the Hausdorff metric. By using this result we obtain elementary proofs of some inequalities, in particular for convolutions and Dirichlet-type integrals, and also the isoperimetric inequality in Euclidean space.

Moreover we derive new symmetry results for minimizers of some variational problems and some properties and relations for general rearrangements along that way.

Furthermore we compare the solutions of two boundary value problems, one of which has a "polarized" geometry, and we show some pointwise inequalities between the solutions. By exploiting the above-mentioned approximation we develop new proofs of functional inequalities which compare the solutions of two elliptic or parabolic problems, one of which has a "Steiner-symmetrized" geometry. The method allows also to investigate the case of equality in the inequalities. Roughly speaking we prove that the original problem has the symmetrized geometry.

\section{BASIC NOTATIONS}

Let $\mathbb{R}^{n}$ be the Euclidean $n$-space and $n \geq 2$. If $x \in \mathbb{R}^{k}, 1 \leq k \leq n$, let $|x|$ be the Euclidean norm of $x$. For $A \subset \mathbb{R}^{n}$ let $\bar{A}$ and $\partial A$ denote the closure and the boundary of $A$, respectively. For $A, B \subset \mathbb{R}^{n}$ let $A+B=\{a+b: a \in A, b \in B\}$, and for $r \in \mathbb{R}$ let $r A=\{r x: x \in A\}$.

We denote by $B_{r}(x)$ the open ball in $\mathbb{R}^{n}$ with radius $r$ and center in $x$, and we write $B_{r}=B_{r}(0)$. If $A \subset \mathbb{R}^{n}$ and $r>0$, then we denote by $A_{r}=A+r \overline{B_{1}}$ and $A_{-r}=A \backslash\left(\partial A+r B_{1}\right)$ the exterior and interior parallel sets of $A$, respectively. Let us recall the following well-known properties (see e.g. [Ha, pp. 147]). For any 
$r, s \geq 0$

$$
\begin{aligned}
& A_{r} \subset A_{s} \quad \text { if } \quad 0 \leq r \leq s, \\
& \left(A_{r}\right)_{s}=A_{r+s}, \quad\left(A_{-r}\right)_{-s}=A_{-r-s}, \\
& \left(A_{-s}\right)_{r} \subset A_{r-s} \subset\left(A_{r}\right)_{-s} .
\end{aligned}
$$

Let $\mathcal{F}$ and $\mathcal{G}$ denote the collection of all nonempty sets in $\mathbb{R}^{n}$ which are compact or open, respectively. Let $\mathcal{G}_{b}$ denote the subclass of $\mathcal{G}$ consisting of all bounded open sets. We define the Hausdorff distance between two compact sets $M, N$ by

$$
d(M, N):=\inf \left\{r>0 ; M \subset N_{r}, N \subset M_{r}\right\} .
$$

It is well known that $d$ is a metric on $\mathcal{F}$ (see [Ha, pp.151]). We call linear submanifolds of $\mathbb{R}^{n}$ planes. Also we will call a point $x \in \mathbb{R}^{n}$ a 0 -dimensional plane through $x$.

We denote by $\mathcal{M}$ the collection of all Lebesgue measurable sets in $\mathbb{R}^{n}$ with finite measure. If $M$ is a measurable set in $\mathbb{R}^{k}(1 \leq k \leq n)$, then $\mathcal{L}^{k}(M)$ denotes its $k$ dimensional measure. Often we treat measurable sets only in a.e. sense, i.e. we write

$$
M=N \quad \text { iff } \quad \mathcal{L}^{k}(M \Delta N)=0
$$

(here $M \Delta N$ denotes the symmetric difference $(M \backslash N) \cup(N \backslash M))$ and

$$
M \subset N \quad \text { iff } \quad \mathcal{L}^{k}(M \backslash N)=0 .
$$

We denote by $\|\cdot\|_{p}$ the usual norm in the space $L^{p}\left(\mathbb{R}^{n}\right), 1 \leq p \leq+\infty$. For functions $u \in C\left(\mathbb{R}^{n}\right)$ we define the modulus of continuity by

$$
\omega_{u}(\delta):=\sup \{|u(x)-u(y)|:|x-y|<\delta\}, \quad \delta>0 .
$$

If $\Omega$ is an open set in $\mathbb{R}^{n}$, we denote by $W^{1, p}(\Omega)$ the Sobolev space of functions $u \in L^{p}(\Omega)$ having generalized partial derivatives $u_{x_{i}} \in L^{p}(\Omega), i=1, \ldots, n$, and we denote by $W_{0}^{1, p}(\Omega)$ the completion of $C_{0}^{\infty}(\Omega)$ in the space $W^{1, p}(\Omega)$. Usually we extend measurable functions $u: \Omega \rightarrow \mathbb{R}_{0}^{+}$by zero outside $\Omega$, so that $W_{0}^{1, p}(\Omega) \subset$ $W^{1, p}\left(\mathbb{R}^{n}\right)$ in that sense (see $[\mathrm{A}]$ ).

By $C_{0}^{0,1}(\Omega)$ we denote the space of Lipschitzean functions with compact support in $\Omega$.

For any function space the lower index "+" denotes the corresponding subspace of nonnegative functions, e.g. $L_{+}^{p}\left(\mathbb{R}^{n}\right), W_{0+}^{1, p}(\Omega), C_{0+}^{0,1}(\Omega), \ldots$.

The space of measurable functions with bounded variation is denoted by $B V\left(\mathbb{R}^{n}\right)$ and we write

$$
\|D u\|_{B V}:=\sup \left\{\int_{\mathbb{R}^{n}} u \sum_{i=1}^{n} \frac{\partial \psi_{i}}{\partial x_{i}} d x: \sum_{i=1}^{n} \psi_{i}^{2} \leq 1, \psi_{i} \in C_{0}^{\infty}\left(\mathbb{R}^{n}\right), i=1, \ldots, n\right\} .
$$

Recall also that if $u \in W^{1,1}\left(\mathbb{R}^{n}\right)$, then $\|D u\|_{B V}=\|\nabla u\|_{1}$. Furthermore, if $M$ is a Caccioppoli set in $\mathbb{R}^{n}$, then $\|D \chi(M)\|_{B V}$ is the perimeter of $M$ in the sense of De Giorgi (see [Ta3]).

Finally, a function $j: \mathbb{R}_{0}^{+} \longrightarrow \mathbb{R}_{0}^{+}$is called a Young function if $j$ is continuous and convex with $j(0)=0$. 


\section{REARRANGEMENTS}

Here we introduce the concept of rearrangement and investigate some general properties which will be of later use.

Any mapping $T: \mathcal{A} \longrightarrow \mathcal{B}$, where $\mathcal{A}$ and $\mathcal{B}$ are nonempty collections of subsets of $\mathbb{R}^{n}$, is called a set transformation. We write $\operatorname{Dom}(T)$ for $\mathcal{A}$ and $\operatorname{Im}(T)$ for the family of image sets $T(A), A \in \mathcal{A}$. If $T_{i}, i=1,2, \ldots, m$, are set transformations such that $\operatorname{Im}\left(T_{i}\right) \subset \operatorname{Dom}\left(T_{i+1}\right), i=1,2, \ldots, m-1$, then $\bigcirc_{i=1}^{m} T_{i}$ denotes their composition $T_{m} \circ \cdots \circ T_{2} \circ T_{1}$.

A set transformation $T$ is called monotone if $T(A) \subset T(B)$ whenever $A, B \in \mathcal{A}$ and $A \subset B$. We say that $T$ is measure preserving if $(\mathcal{F} \cup \mathcal{G}) \cap \mathcal{M} \subset \mathcal{A}$ and $\mathcal{L}^{n}(T(M))=\mathcal{L}^{n}(M)$ for all sets $M \in(\mathcal{F} \cup \mathcal{G}) \cap \mathcal{M}$. Every monotone and measure preserving set transformation is called a rearrangement.

If $T$ is a rearrangement, there exists a continuation $\tilde{T}$ of $T$ onto $\mathcal{M}$, which is again a rearrangement. (Obviously the images $\tilde{T}(M), M \in \mathcal{M}$, and the monotonicity of $\tilde{T}$ have to be understood in the a.e. sense (2.4) and (2.5)!) It is easy to verify that $\tilde{T}$ is uniquely determined, and if $M \in \mathcal{M}$, then

$$
\tilde{T}(M)=\bigcap_{i=1}^{\infty} T\left(G_{i}\right)
$$

for every decreasing sequence $\left\{G_{i}\right\} \subset \mathcal{G}$ with $\lim _{i \rightarrow \infty} \mathcal{L}^{n}\left(G_{i} \backslash M\right)=0$. Also

$$
\tilde{T}(M)=\bigcup_{i=1}^{\infty} T\left(F_{i}\right)
$$

for every increasing sequence $\left\{F_{i}\right\} \subset \mathcal{F}$ with $\lim _{i \rightarrow \infty} \mathcal{L}^{n}\left(M \backslash F_{i}\right)=0$.

Of course we will treat the transformation $T$ and its natural continuation $\tilde{T}$ as the same rearrangement. Nevertheless in some cases we have to distinguish strictly between the different definitions of $T$ in the classes $\mathcal{F} \cup \mathcal{G}$ and $\mathcal{M}$.

Now we introduce a natural class of functions for which a rearrangement can be defined. We say that a measurable function $u: \mathbb{R}^{n} \longrightarrow \mathbb{R}$ belongs to $\mathcal{S}$ ( the class of "symmetrizable" functions ) if

$$
\mathcal{L}^{n}(\{u>c\})<+\infty \quad \forall c>\inf u .
$$

(Here and in the following we use the abbreviation $\{u>c\}=\left\{x \in \mathbb{R}^{n}: u(x)>c\right\}$.) Note that the spaces $L_{+}^{p}\left(\mathbb{R}^{n}\right), W_{+}^{1, p}\left(\mathbb{R}^{n}\right)(1 \leq p<+\infty)$, and $C_{0+}^{0,1}\left(\mathbb{R}^{n}\right)$ are subspaces of $\mathcal{S}_{+}$.

If $T$ is a rearrangement and $u$ is a continuous function in $\mathcal{S}$, then the relations

$$
T u(x):=\sup \{c>\inf u: x \in T(\{u>c\})\} \quad x \in \mathbb{R}^{n}, \quad \inf T u:=\inf u,
$$

define a function $T u$. If $u$ is in $\mathcal{S}$ but is not continuous, we define $T u$ by replacing "sup" in (3.1) by "ess sup". Clearly the function $T u$ is uniquely determined almost everywhere.

From (3.1) one obtains

$$
\bigcup_{\mu>c} \bigcap_{\lambda<\mu} T(\{u>\lambda\}) \subset\{T u>c\} \subset T(\{u>c\}) \quad \forall c>\inf u .
$$


Since $T$ is measure preserving, this means that

$$
\mathcal{L}^{n}(T(\{u>c\}))=\mathcal{L}^{n}(\{T u>c\}) \quad \forall c>\inf u .
$$

Thus the function $T u$ also belongs to $\mathcal{S}$. In this way we have constructed a mapping $T: \mathcal{S} \longrightarrow \mathcal{S}$, which we call again a rearrangement.

In the special case that $u$ is a characteristic function, i.e. $u=\chi(M), M \in \mathcal{M}$, (3.1) simply reduces to

$$
T(\chi(M))=\chi(T(M)),
$$

which shows that every rearrangement for sets can be "reconstructed" from the corresponding rearrangement for functions. The monotonicity of $T$ for functions reads as

$$
u, v \in \mathcal{S}, u \leq v \Longrightarrow T u \leq T v,
$$

from which we obtain the following property:

$$
u \in \mathcal{S}, \varphi \text { nondecreasing } \Longrightarrow \varphi(u) \in \mathcal{S} \text { and } \varphi(T u)=T(\varphi(u)) .
$$

Further on it is well known (see e.g. [Ka1]) that one can deduce "Cavalieri's principle" from (3.3) and (3.5)

$$
\int_{\mathbb{R}^{n}} f(u) d x=\int_{\mathbb{R}^{n}} f(T u) d x \quad \forall u \in \mathcal{S}, \forall \text { continuous } f,
$$

whenever either one of the integrals in (3.7) converges.

The following theorem (for a proof see [CZR Theorem 3, Corollary 1]) will be very useful for approximations of the rearranged functions:

Theorem 3.1 (Nonexpansivity of rearrangements). Let $T$ be a rearrangement. Then we have for every Young function $j$

$$
\int_{\mathbb{R}^{n}} j(|T u-T v|) d x \leq \int_{\mathbb{R}^{n}} j(|u-v|) d x \quad \forall u, v \in \mathcal{S},
$$

whenever either one of the integrals in (3.8) converges.

Remark 3.1. In the special case $j(t)=t^{p},(1 \leq p \leq+\infty)$, one concludes from (3.8) the nonexpansivity in $L^{p}\left(\mathbb{R}^{n}\right)$

$$
\|T u-T v\|_{p} \leq\|u-v\|_{p} \quad \forall u, v \in L_{+}^{p}\left(\mathbb{R}^{n}\right) .
$$

Furthermore, (3.8) with $p=2$ and (3.7) yield the well-known Hardy-Littlewood inequality

$$
\int_{\mathbb{R}^{n}} u v d x \leq \int_{\mathbb{R}^{n}} T u T v d x \quad \forall u, v \in L_{+}^{2}\left(\mathbb{R}^{n}\right) .
$$

If $u \in \mathcal{S}$, the distribution function $m_{u}$ is given by

$$
m_{u}(c):=\mathcal{L}^{n}(\{u>c\}), \quad c>\inf u .
$$

Sometimes we will say that two functions $u, v \in \mathcal{S}$ are rearrangements of each other if $\inf u=\inf v$ and $m_{u}(c)=m_{v}(c) \forall c>\inf u$. 
Let us add definitions of some further properties of set transformations.

A set transformation $T: \mathcal{A} \rightarrow \mathcal{B}$ is called open or compact if $\mathcal{G} \subset \mathcal{A}$ or $\mathcal{F} \subset \mathcal{A}$ and $T(A)$ is open or compact whenever $A$ is of the same kind, respectively. We say that $T$ is continuous from the inside if $\bigcup_{i} T\left(G_{i}\right)=T\left(\bigcup_{i} G_{i}\right)$ for every increasing sequence $\left\{G_{i}\right\} \subset \mathcal{G}$. Similarly we say that $T$ is continuous from the outside if $\bigcap_{i} T\left(F_{i}\right)=T\left(\bigcap_{i} F_{i}\right)$ for every decreasing sequence $\left\{F_{i}\right\} \subset \mathcal{F}$.

Finally a rearrangement $T$ is called smoothing if

$$
T\left(F_{r}\right) \supset(T(F))_{r}
$$

for every $F \in \mathcal{F}$ and $r>0$.

Smoothing rearrangements were introduced by Sarvas [Sa].

Lemma 3.1. Let $T$ be a rearrangement which is continuous from the inside and let $u \in C\left(\mathbb{R}^{n}\right) \cap \mathcal{S}$. Then

$$
\{T u>c\}=T(\{u>c\}) \quad \forall c>\inf u .
$$

Proof. Let $c>\inf u$. Since $u$ is continuous, all the level sets $\{u>\lambda\}$ with $\lambda>\inf u$ are open. Together with the continuity from inside this yields

$$
T(\{u>c\})=\bigcup_{i=1}^{\infty} T\left(\left\{u>c+\frac{1}{i}\right\}\right)
$$

Now let $x \in T(\{u>c\})$. By (3.1) this means that $T u(x) \geq c$.

Assume first that $T u(x)=c$. Then $x$ cannot belong to any of the level sets $T(\{u>\lambda\}), \lambda>c$. But this impossible by (3.13).

It follows that $T u(x)>c$. Thus we have proved that $T(\{u>c\}) \subset\{T u>c\}$. Now the assertion follows in view of (3.2).

The next lemma was shown in $\underline{\mathrm{Sa}}$.

Lemma 3.2. Let $T$ be a monotone and smoothing set transformation which is continuous from the inside and let $(\mathcal{F} \cup \mathcal{G}) \subset$ Dom $T$. Then $T$ is open.

Lemma 3.3. Let $T$ be a monotone and smoothing set transformation which is continuous from the outside and let $(\mathcal{F} \cup \mathcal{G}) \subset$ Dom $T$. Then $T$ is compact.

Proof. Let $F \in \mathcal{F}$. By the continuity from the outside we have $\bigcap_{i} T\left(F_{(1 / i)}\right)=T(F)$. Since $T$ is smoothing, we conclude that also $\bigcap_{i}\left(T(F)_{(1 / i)}\right)=T(F)$. But this means that $T(F)$ is compact.

Lemma 3.4. Let $T$ be as in Lemma 3.3. Then

$$
\begin{aligned}
& T\left(G+r B_{1}\right) \supset T(G)+r B_{1}, \\
& T\left(G \backslash(\partial G)_{r}\right) \subset T(G) \backslash(\partial T(G))_{r} \quad \forall G \in \mathcal{G}, r>0,
\end{aligned}
$$

and

$$
\operatorname{dist}\{M ; \partial N\} \leq \operatorname{dist}\{T(M) ; \partial T(N)\} \quad \forall M, N \in \mathcal{G} \text { with } M \subset N .
$$


Proof. Let us assume that $G \in \mathcal{G}_{b}$. Then for $r>\varepsilon>0$ we have

$$
G+r B_{1} \supset \bar{G}_{(r-\varepsilon)} \text {. }
$$

Since $T$ is monotone and smoothing, this means that

$$
T\left(G+r B_{1}\right) \supset T\left(\bar{G}_{(r-\varepsilon)}\right) \supset(T(\bar{G}))_{(r-\varepsilon)} \supset T(G)+(r-\varepsilon) B_{1} .
$$

By passing to the limit $\varepsilon \searrow 0$, we derive (3.14) in this case.

If $G$ is unbounded, we choose an increasing sequence $\left\{G_{i}\right\} \subset \mathcal{G}_{b}$ with $\bigcup_{i=1}^{\infty} G_{i}=$ $G$. Then by the monotonicity we have

$$
T\left(G+r B_{1}\right) \supset T\left(G_{i}+r B_{1}\right) \supset T\left(G_{i}\right)+r B_{1}, \quad i=1,2, \ldots .
$$

Since $T$ is continuous from the inside, we can pass to the limit for $i \rightarrow+\infty$ and obtain (3.14).

Next set $r:=\operatorname{dist}\{M ; \partial N\}$. Then by the monotonicity and (3.14) we conclude that

$$
T(N) \supset T\left(M+r B_{1}\right) \supset T(M)+r B_{1},
$$

which yields (3.16).

Finally, by setting $N=G$ and $M=G \backslash(\partial G)_{r}$ in (3.16), we derive

$$
r \leq \operatorname{dist}\left\{T\left(G \backslash(\partial G)_{r}\right) ; \partial T(G)\right\}
$$

which is (3.15).

Because of the well-known Brunn-Minkowski inequality it is not surprising that the smoothing property implies that the rearrangement transforms balls into balls. More precisely the following holds.

Theorem 3.2. Let $T$ be a smoothing rearrangement which is continuous from the outside and from the inside. Then for each $x_{0} \in \mathbb{R}^{n}$ there is some $y_{0} \in \mathbb{R}^{n}$ such that

$$
\begin{aligned}
& T\left(B_{r}\left(x_{0}\right)\right)=B_{r}\left(y_{0}\right) \text { and } \\
& T\left(\overline{B_{r}\left(x_{0}\right)}\right)=\overline{B_{r}\left(y_{0}\right)} \quad \forall r>0 .
\end{aligned}
$$

Proof. For $G \in \mathcal{G}_{b}$ we set $\delta(G):=\sup \{\operatorname{dist}\{x ; \partial G\}: x \in G\}$. Let $x_{0} \in \mathbb{R}^{n}$ and $R>0$ and assume that $T\left(B_{R}\left(x_{0}\right)\right)$ is not a ball. Then we have $\delta\left(T\left(B_{R}\left(x_{0}\right)\right)\right)<R-\varepsilon$ for some $\varepsilon>0$. It follows that

$T\left(B_{\varepsilon}\left(x_{0}\right)\right)=T\left(B_{R}\left(x_{0}\right) \backslash\left(\partial B_{R}\left(x_{0}\right)\right)_{R-\varepsilon}\right) \subset T\left(B_{R}\left(x_{0}\right)\right) \backslash\left(\partial T\left(B_{R}\left(x_{0}\right)\right)\right)_{R-\varepsilon}=\emptyset$, which contradicts the equimeasurability. Thus there is some $y_{0} \in \mathbb{R}^{n}$, such that $T\left(B_{R}\left(x_{0}\right)\right)=B_{R}\left(y_{0}\right)$. Next let $R>r>0$. Then, by (3.16), we have

$$
\operatorname{dist}\left\{T\left(B_{r}\left(x_{0}\right)\right) ; B_{R}\left(x_{0}\right)\right\} \geq R-r,
$$

which is (3.17).

Finally (3.18) follows by approximation of $\overline{B_{r}\left(x_{0}\right)}$ with open balls and from the fact that $T$ is compact.

Theorem 3.3. Let $T$ be a rearrangement which is continuous from the inside. Then

$$
\begin{aligned}
& T \quad \text { is smoothing } \Longleftrightarrow \\
& \omega_{T u} \leq \omega_{u} \quad \forall u \in C\left(\mathbb{R}^{n}\right) \cap \mathcal{S} .
\end{aligned}
$$


Proof. 1) Let $T$ be smoothing. By Lemma 3.3 all the level sets $\{T u>c\}, c>\inf u$, are open. Applying (3.16) leads to

$$
\begin{aligned}
& 0<\operatorname{dist}\left\{\left\{u>c_{2}\right\} ; \partial\left\{u>c_{1}\right\}\right\} \leq \operatorname{dist}\left\{\left\{T u>c_{2}\right\} ; \partial\left\{T u>c_{1}\right\}\right\} \\
& \forall c_{2}>c_{1}>\inf u,
\end{aligned}
$$

which means that $T u$ is continuous.

Now let $\delta>0$. There are points $x_{1}, x_{2}$ with $\left|x_{1}-x_{2}\right|=\delta$ and

$$
\omega_{T u}(\delta)=\left|T u\left(x_{1}\right)-T u\left(x_{2}\right)\right| .
$$

We can assume that $T u\left(x_{1}\right)=c_{1}<c_{2}=T u\left(x_{2}\right)$. Then $x_{i} \in \partial\left\{T u>c_{i}\right\}, i=1,2$, and thus dist $\left\{\left\{T u>c_{2}\right\} ; \partial\left\{T u>c_{1}\right\}\right\}=\delta$. Now from (3.16) we conclude that $\delta \leq \operatorname{dist}\left\{\left\{u>c_{2}\right\} ; \partial\left\{u>c_{1}\right\}\right\}$, which means that $\omega_{u}(\delta) \geq c_{2}-c_{1}=\omega_{T u}(\delta)$.

2) Now assume that $T$ satisfies (3.19). Let $F \in \mathcal{F}, r>0$ and set

$$
u(x):= \begin{cases}r-\operatorname{dist}\{x ; F\} & \text { if } x \in F_{r}, \\ 0 & \text { if } x \in \mathbb{R}^{n} \backslash F_{r} .\end{cases}
$$

Then clearly $u \in C\left(\mathbb{R}^{n}\right) \cap \mathcal{S}$ and

$$
r=\omega_{u}(r) \geq \omega_{T u}(r) .
$$

In view of $F=\{u=r\}, F_{r}=\operatorname{supp} u, T F=\{T u=r\}$ and $T\left(F_{r}\right)=\operatorname{supp} T u$ this implies that

$$
\operatorname{dist}\left\{T F ; \partial T\left(F_{r}\right)\right\} \geq r \text {, }
$$

and the assertion follows.

Corollary 3.1. Let $T$ be a smoothing rearrangement which is continuous from the inside and let $u \in C\left(\mathbb{R}^{n}\right) \cap \mathcal{S}$ satisfy a Hölder condition with exponent $\alpha \in(0,+1]$ and constant $L$, i.e.

$$
|u(x)-u(y)| \leq L|x-y|^{\alpha} \quad \forall x, y \in \mathbb{R}^{n} .
$$

Then Tu also satisfies a Hölder condition with exponent $\alpha$ and constant less than or equal to $L$.

\section{Steiner Symmetrizations}

Let us now recall the definitions of the Steiner symmetrizations (for further information see [St], $[\mathrm{L}]$ and $[\mathrm{Sa}])$.

Every $(n-k)$-dimensional plane $\Sigma \subset \mathbb{R}^{n}, 1 \leq k \leq n$, defines a $(k, n)$-Steiner symmetrization $S$ as follows:

For every $x \in \Sigma$ let $\Lambda(x)$ denote the $k$-dimensional plane through $x$ and orthogonal to $\Sigma$.

1) Let $M \in(\mathcal{F} \cup \mathcal{G}) \cap \mathcal{M}$. If $\mathcal{L}^{k}(M \cap \Lambda(x))=0$, then $S(M) \cap \Lambda(x)$ is empty or the point $\{x\}$ according to whether $M \cap \Lambda(x)$ is empty or nonempty. If $\mathcal{L}^{k}(M \cap \Lambda(x))>$ 0 , then

$$
S(M) \cap \Lambda(x)= \begin{cases}B_{r}(x) \cap \Lambda(x) & \text { if } M \text { is open, } \\ B_{r}(x) \cap \Lambda(x) & \text { if } M \text { is compact, }\end{cases}
$$

where $r$ is defined by $r>0$ and $\mathcal{L}^{k}\left(B_{r}(x) \cap \Lambda(x)\right)=\mathcal{L}^{k}(M \cap \Lambda(x))$.

2) Let $M \in \mathcal{M}$ but be neither open nor compact. Then the sets $S(M) \cap \Lambda(x)$ are defined in a.e. sense by either one of (4.1). 
From the definition one deduces immediately that the $(k, n)$ - Steiner symmetrization is a rearrangement which is continuous from the inside and from the outside. Note also that in case 2) Fubini's Theorem implies that the sets $M \cap \Lambda(x)$ are measurable with finite $\mathcal{L}^{k}$-measure for a.e. $x \in \Sigma$.

The $(n, n)$-Steiner symmetrization is often called Schwarz symmetrization or symmetric decreasing rearrangement, and we will denote it by $S^{\star}$.

For our purposes it will often be helpful to use a special coordinate system in $\mathbb{R}^{n}$ :

$$
x=\left(x_{1}, \ldots, x_{n}\right)=\left(x^{\prime}, y\right), \quad x^{\prime}=\left(x_{1}, \ldots, x_{n-k}\right), y=\left(x_{n-k+1}, \ldots, x_{n}\right),
$$

in which the plane $\Sigma$ of symmetry becomes simply $\{y=0\}$. If $M \in \mathcal{M}$, we introduce the " $x$ '- slices" of $M$ by

$$
M\left(x^{\prime}\right)=\left\{y \in \mathbb{R}^{k}:\left(x^{\prime}, y\right) \in M\right\}, \quad x^{\prime} \in \mathbb{R}^{n-k} .
$$

Let $S^{\star}\left(M\left(x^{\prime}\right)\right)$ denote the Schwarz symmetrization of $M\left(x^{\prime}\right)$, taken in $\mathbb{R}^{k}$. Then (4.1) reads

$$
S(M):=\left\{x=\left(x^{\prime}, y\right): y \in S^{\star}\left(M\left(x^{\prime}\right)\right), x^{\prime} \in \mathbb{R}^{n-k}\right\} .
$$

If $u \in \mathcal{S}$, then we obtain from (4.1) that the $(k, n)$-Steiner symmetrization $S u$ of $u$ is given by the relations

$$
S u\left(x^{\prime}, y\right)=\sup \left\{c>\inf u: x \in S\left(\left\{u\left(x^{\prime}, \cdot\right)>c\right\}\right)\right\} .
$$

(Here and in the following for simplicity $\left\{u\left(x^{\prime}, \cdot\right)>c\right\}$ denotes $\left\{y \in \mathbb{R}^{k}: u\left(x^{\prime}, y\right)>\right.$ c\}.)

Let us mention again that the equations (4.2) and (4.3) have to be understood in the pointwise sense iff $u$ is continuous. Note also that $S u$ is "radially symmetric and decreasing in $|y|$ ", i.e.

$$
\begin{aligned}
& S u\left(x^{\prime}, y\right)=S u\left(x^{\prime}, z_{1}\right) \geq S u\left(x^{\prime}, z_{2}\right) \\
& \quad \text { if }|y|=\left|z_{1}\right| \leq\left|z_{2}\right|, \quad x^{\prime} \in \mathbb{R}^{n-k}, y, z_{1}, z_{2} \in \mathbb{R}^{k} .
\end{aligned}
$$

Sometimes we will also write $S(M)=M^{*}$ and $S u=u^{*}$ for the symmetrized objects.

\section{Polarization}

Let $\Sigma$ be some $(n-1)$-dimensional affine hyperplane in $\mathbb{R}^{n}$ and assume that $H$ is one of the open halfspaces into which $\mathbb{R}^{n}$ is subdivided by $\Sigma$. Let $\sigma_{H}$ denote the reflection in $\Sigma$. We write $\bar{x}=\sigma_{H}(x)$ for points $x \in \mathbb{R}^{n}$ and $\sigma_{H}(u)(x)=u(\bar{x}) \forall x \in$ $\mathbb{R}^{n}$ for functions $u \in \mathcal{S}$.

Definition 5.1. If $u \in \mathcal{S}$, then its polarization $P u$ (with respect to $H$ ) is given by

$$
P u(x):= \begin{cases}\max \{u(x) ; u(\bar{x})\} & \text { if } x \in H, \\ \min \{u(x) ; u(\bar{x})\} & \text { if } x \in \mathbb{R}^{n} \backslash H .\end{cases}
$$

If $M \in \mathcal{M}$, then the polarization $P(M)$ is given by its characteristic function via (5.1), i.e.

$$
\chi(P(M)):=P(\chi(M)) .
$$


In the case that $u$ is continuous and $M$ is open or closed, equations (5.1) and (5.2) have to be understood in the pointwise sense.

Equations (5.1) and (5.2) could also be written in the following more precise form

$$
P(M)=\left(\left(M \cup \sigma_{H}(M)\right) \cap H\right) \cup\left(M \cap \sigma_{H}(M)\right), \quad M \in \mathcal{M},
$$

and

$$
P u(x)=\sup \{c>\inf u: x \in P(\{u>c\})\}, \quad x \in \mathbb{R}^{n}, \quad u \in \mathcal{S} .
$$

From the representations (5.1)-(5.4) we see that the polarization $P$ is an open and compact rearrangement which is continuous from the inside and from the outside.

For the sake of simplicity, we will often use the subscript " $H$ " to denote any one of the polarized objects, i.e. we write $u_{H}$ and $M_{H}$ for $P u$ and $P(M)$, respectively.

The following lemma was proved in Bae2 p.58]. (For a proof of a similar property in the case of the sphere $S^{n}$ compare also [BT, Lemma 1]). Together with Theorem 3.3 it shows that polarization is a smoothing rearrangement.

Lemma 5.1. Let $u \in C\left(\mathbb{R}^{n}\right) \cap \mathcal{S}$ and let $u_{H}$ be some polarization of $u$. Then

$$
\omega_{u_{H}} \leq \omega_{u}
$$

The following lemma shows that the polarization depends continuously on its defining halfspace (see also $[\mathrm{Br}]$ ).

Lemma 5.2. Let $u \in L_{+}^{p}\left(\mathbb{R}^{n}\right),(1 \leq p<+\infty)$, and let $\left\{H_{m}\right\}$ be a sequence of halfspaces.

1) If $H$ is a halfspace and

$$
\lim _{m \rightarrow \infty} \mathcal{L}^{n}\left(\left(H_{m} \Delta H\right) \cap B_{R}\right)=0 \quad \forall R>0,
$$

then

$$
u_{H_{m}} \longrightarrow u_{H} \quad \text { in } \quad L^{p}\left(\mathbb{R}^{n}\right) .
$$

2) If $B_{R_{m}} \subset H_{m}, m=1,2, \ldots$, for some sequence $R_{m} \nearrow+\infty$, then

$$
u_{H_{m}} \longrightarrow u \quad \text { in } L^{p}\left(\mathbb{R}^{n}\right) \text {. }
$$

Proof. 1) By (5.6) we have

$$
\lim _{m \rightarrow \infty} \sigma_{H_{m}}(x)=\sigma_{H}(x), \quad \text { uniformly in compact subsets of } \mathbb{R}^{n} .
$$

This leads to (5.7) in case that $u$ is continuous with compact support.

In the general case let $\varepsilon>0$. We choose a continuous function $v$ with compact support such that $\|u-v\|_{p}<\varepsilon / 3$, and then $m_{0}$ large enough such that

$$
\left\|v_{H_{m}}-v_{H}\right\|_{p}<\frac{\varepsilon}{3} \quad \forall m \geq m_{0}
$$

Applying Lemma 3.1 we obtain

$$
\begin{aligned}
\left\|u_{H_{m}}-u_{H}\right\|_{p} & \leq\left\|u_{H_{m}}-v_{H_{m}}\right\|_{p}+\left\|v_{H_{m}}-v_{H}\right\|_{p}+\left\|v_{H}-u_{H}\right\|_{p} \\
& \leq 2\|u-v\|_{p}+\left\|v_{H_{m}}-v_{H}\right\|_{p}<\varepsilon \quad \forall m \geq m_{0},
\end{aligned}
$$

and (5.7) follows.

2) If $u$ has bounded support, there is some $m_{0} \in \mathbb{N}$ such that

$$
u_{H_{m}}=u \quad \forall m \geq m_{0} .
$$

In the general case we argue similarly as in part 1) to derive (5.8). 
It was observed by Dubinin [Du1] Du3 that certain capacities decrease under polarization because the Dirichlet norms $\|\nabla u\|_{p}$ do not change under polarization if the competing functions are sufficiently smooth. The following lemma shows that this property remains true even in Sobolev-spaces $W^{1, p}\left(\mathbb{R}^{n}\right)$.

Lemma 5.3. Let $u \in W_{+}^{1, p}\left(\mathbb{R}^{n}\right)(1 \leq p \leq+\infty)$, and let $H$ be some halfspace. Then $u_{H} \in W_{+}^{1, p}\left(\mathbb{R}^{n}\right)$ and $|\nabla u|$ and $\left|\nabla u_{H}\right|$ are rearrangements of each other. Furthermore, if $\vec{n}$ is the unit normal to the hyperplane $\partial H, V$ is some linear subspace, which either contains $\vec{n}$ or is orthogonal to $\vec{n}$, and $\nabla_{V}$ is the gradient with respect to $V$, then $\left|\nabla_{V} u\right|$ and $\left|\nabla_{V} u_{H}\right|$ are rearrangements of each other too. In particular we have

$$
\left\|\nabla u_{H}\right\|_{p}=\|\nabla u\|_{p}
$$

and

$$
\left\|\nabla_{V}\left(u_{H}\right)\right\|_{p}=\left\|\nabla_{V} u\right\|_{p}
$$

Proof. We set

$$
v(x):=u(\bar{x}), w(x):=u_{H}(\bar{x}), \quad x \in H .
$$

Since $u_{H}(x)=\max \{u(x) ; v(x)\}=v(x)+(u(x)-v(x))+$ and $w(x)=\min \{u(x) ; v(x)\}$ $=u(x)-(u(x)-v(x))$ for all $x \in H$, we can conclude (compare GT, Lemma 7.6, p.152]) that $u_{H}, w \in W^{1,1}(H)$ and

$$
\begin{aligned}
\nabla u_{H}(x) & = \begin{cases}\nabla u(x) & \text { a.e. on }\{u>v\} \cap H, \\
\nabla v(x) & \text { a.e. on }\{u \leq v\} \cap H,\end{cases} \\
\nabla w(x) & = \begin{cases}\nabla v(x) & \text { a.e. on }\{u>v\} \cap H, \\
\nabla u(x) & \text { a.e. on }\{u \leq v\} \cap H .\end{cases}
\end{aligned}
$$

From these formulas the assertions follow immediately.

Corollary 5.1. Let $\Omega \subset \mathbb{R}^{n}$ be an open set, let $H$ be a halfspace and $u \in W_{0+}^{1, p}(\Omega)$ $(1 \leq p<+\infty)$. Then we have $u_{H} \in W_{0+}^{1, p}\left(\Omega_{H}\right)$ and (5.9), (5.10) hold.

Proof. The formulas (5.9) and (5.10) follow from Lemma 5.3 by extending $u$ by zero outside $\Omega$. It remains to show that $u_{H} \in W_{0}^{1, p}\left(\Omega_{H}\right)$.

Assume that $u \in C_{0+}^{0,1}(\Omega)$. In view of Lemma 5.1 and Theorem 3.3 this means that $u_{H} \in C_{0+}^{0,1}\left(\Omega_{H}\right)$.

In the general case we choose a sequence $u_{m}$ of functions in $C_{0+}^{0,1}(\Omega)$ which converges to $u$ in $W_{0}^{1, p}(\Omega)$. Then $\left(u_{m}\right)_{H} \rightarrow u_{H}$ in $L^{2}\left(\Omega_{H}\right)$. The functions $\left(u_{m}\right)_{H}$ are equibounded in $W_{0}^{1, p}\left(\Omega_{H}\right)$ by Lemma 5.3. Hence we find a function $v \in W_{0}^{1, p}\left(\Omega_{H}\right)$ and a subsequence $\left(u_{m^{\prime}}\right)_{H}$ which converges to $v$ weakly in $W_{0}^{1, p}\left(\Omega_{H}\right)$. This means that for every $\varphi \in C_{0}^{\infty}\left(\Omega_{H}\right)$ and $i \in\{1, \ldots, n\}$

$$
\begin{aligned}
\int_{\Omega_{H}} \varphi v_{x_{i}} d x \longleftarrow & \int_{\Omega_{H}} \varphi \frac{\partial\left(\left(u_{m^{\prime}}\right)_{H}\right)}{\partial x_{i}} d x \\
& =-\int_{\Omega_{H}} \varphi_{x_{i}}\left(u_{m^{\prime}}\right)_{H} d x \longrightarrow-\int_{\Omega_{H}} \varphi_{x_{i}} u_{H} d x
\end{aligned}
$$

that is, $v=u_{H}$. The corollary is proved. 
Lemma 5.3 has some easy consequences in symmetry problems.

Consider a variational problem of the following form:

$$
\text { (P) } J(v) \equiv \int_{\Omega}\left(\frac{1}{p}|\nabla v|^{p}-F(x, v)\right) d x \longrightarrow \text { Min !, } \quad v \in K,
$$

where $\Omega$ is a bounded domain in $\mathbb{R}^{n}, K$ is a closed subset of $W_{0}^{1, p}(\Omega),(1<p<+\infty)$, and $F=F(x, w)$ is defined on $\Omega \times \mathbb{R}$, bounded and measurable in $x$ for a.e. $w \in \mathbb{R}$ and continuous in $w$.

We shall not discuss the existence and uniqueness of solutions to problem $(\mathbf{P})$. We are interested in symmetry properties of the minimizers under the assumption that the problem has a unique positive solution.

Remark 5.1. 1) Positive minimizers of problems like (P) may describe stable (ground) states of equilibria in plasma physics, heat conduction and chemical reactors (see e.g. [Di, section 4]).

2) Let us briefly recall the connection to boundary value problems in some wellknown special cases.

a) If $K=W_{0}^{1, p}(\Omega)$ and $F(x, w)$ is differentiable in $w$, then a minimizer $u$ of $(\mathbf{P})$ is a weak solution of the boundary value problem

$$
u \in W_{0}^{1, p}(\Omega), \quad-\nabla\left(|\nabla u|^{p-2} \nabla u\right)=F_{u}(x, u) \quad \text { in } \Omega .
$$

b) If $K=W_{0}^{1, p}(\Omega)$ and $F(x, w)$ is concave in $w$, then a minimizer $u$ of $(\mathbf{P})$ is a weak solution of the following differential inclusion:

$$
u \in W_{0}^{1, p}(\Omega), \quad-\nabla\left(|\nabla u|^{p-2} \nabla u\right) \in f(x, u) \quad \text { in } \Omega,
$$

where $f$ denotes the maximal monotone graph of $(\partial F) /(\partial w)$, i.e.

$$
f(x, w):=\left[\liminf _{v \rightarrow w} \frac{F(x, v)-F(x, w)}{v-w}, \limsup _{v \rightarrow w} \frac{F(x, v)-F(x, w)}{v-w}\right], x \in \Omega, w \in \mathbb{R} .
$$

Theorem 5.1. Let $K \subset W_{0+}^{1, p}(\Omega)$ and let problem $(\boldsymbol{P})$ have a unique minimizer $u$. Assume that, if $v, w \in W_{0+}^{1, p}(\Omega), v \in K$, and $v, w$ are rearrangements of each other, then we have also $w \in K$. Furthermore assume that there is some halfspace $H$ such that $\Omega=\Omega_{H}$ and

$$
F(x, v)-F(\bar{x}, v) \leq F(x, w)-F(\bar{x}, w), \quad \text { if } \quad 0 \leq v \leq w, x \in \Omega \cap H .
$$

Then

$$
u=u_{H} .
$$

Proof. By (5.14) we have

$$
F\left(x, u_{H}(x)\right)+F\left(\bar{x}, u_{H}(\bar{x})\right) \geq F(x, u(x))+F(\bar{x}, u(\bar{x})) \quad \forall x \in \Omega \cap H .
$$

By integrating this inequality over $\Omega \cap H$ and then taking Lemma 5.3 into account, we conclude immediately.

At this place let us refer to one simple geometric observation which motivates our approach in the next sections. 
If a function $u$ is equal to its polarization $u_{H}$ for a whole continuum of halfspaces $H$, then $u$ satisfies some monotonicity (or even symmetry) properties. In particular one can identify "symmetrized" functions in this way (see Lemma 6.3).

Next we will study situations in which we can obtain symmetries of the solutions of (P) by using Theorem 5.1.

We fix a decomposition $\mathbb{R}^{n} \ni x=\left(x^{\prime}, y\right), y \in \mathbb{R}$. Let $H_{t}$ denote the halfspace $\{y>t\}, t \in \mathbb{R}$, and let “*” denote the $(1, n)$-Steiner symmetrization with respect to $\{y=0\}$.

Corollary 5.2. Let $\Omega, K, u$ as in Theorem 5.1. We consider two cases.

1) Suppose that (i) and (ii) hold.

(i) There is a number $t \in \mathbb{R}$, such that $\Omega=\Omega_{H_{s}} \forall s \leq t$;

(ii) for all numbers $v, w$ with $0 \leq v \leq w$, the function $\varphi(x):=F(x, w)-$ $F(x, v), x \in \Omega$, is monotonically nondecreasing in $y$ for $y \leq t$, and condition (5.14) is satisfied with $H=H_{t}$.

Then $u$ is monotonically nondecreasing in $y$ for $y \leq t$ and $u=u_{H_{t}}$.

2) Let $\Omega=\Omega^{*}$ and assume that for all numbers $v, w$ with $0 \leq v \leq w$, the function $\varphi(x)$ defined in 1) is monotonically nondecreasing in $y$ for $y \leq 0$ and condition (5.14) is satisfied with the equality sign for $H=H_{0}$. Then $u=u^{*}$.

Proof. 1) By applying Theorem 5.1 we have that

$$
u=u_{H_{s}} \quad \forall s \leq t,
$$

from which we easily obtain the first assertion.

2) In view of the assumption we conclude that $J(u)=J\left(\sigma_{H_{0}}(u)\right)$. By uniqueness this means that $u=\sigma_{H_{0}}(u)$, and the second assertion follows by applying part 1).

Remark 5.2. 1) Some special cases of Corollary 5.2 are well-known if $F(x, w)$ is differentiable in $w$ or is independent of $x$ (see e.g. Ka1, p. $78 \mathrm{ff}$.]). If $F$ is differentiable in $w$, then the second condition (ii) in part 1) means that

$$
\begin{aligned}
& \frac{\partial F}{\partial w}\left(x^{\prime}, y, w\right) \quad \text { is monotonically nondecreasing in } y \text { and } \\
& \frac{\partial F}{\partial w}\left(x^{\prime}, y, w\right) \leq \frac{\partial F}{\partial w}\left(x^{\prime}, 2 t-y, w\right) \quad \forall\left(x^{\prime}, y\right) \in \Omega \text { with } y \leq t, \forall w \in \mathbb{R}_{0}^{+},
\end{aligned}
$$

and part 2) can equivalently be written as

$$
\begin{gathered}
\Omega=\Omega^{*} \text { and } \frac{\partial F}{\partial w}\left(x^{\prime}, y, w\right) \text { is symmetric in } y \text { and monotonically nondecreasing } \\
\quad \text { in } y \text { for } y \leq 0, \quad\left(x^{\prime}, y, w\right) \in \Omega \times \mathbb{R}_{0}^{+} .
\end{gathered}
$$

2) If $p=2$ and $\frac{\partial F}{\partial w}(x, w)$ is Lipschitz continuous in $w$, then one can prove the same symmetry results as in Theorem 5.1 and Corollary 5.2, even in the case that $u$ is no longer a minimizer of $(\mathbf{P})$, but is only a positive weak solution of the problem (5.12). The proof which then establishes the symmetry is based on the so-called moving plane method. (For important references we mention the papers $[\mathrm{GNN},[\mathrm{BN}]$ and [Da], where many related symmetry results were obtained.)

Remark 5.3. Let $n=2, M=\left\{x=\left(x_{1}, x_{2}\right): x_{1} \in[0,1], x_{2} \in[-2,-1] \cup[0,1]\right\}$ and $H$ be the halfspace $\left\{x_{2}>-1\right\}$. Then $M_{H}=[0,1] \times[-1,+1]$. This example shows that the polarization may decrease the surface area of compact sets with sufficiently smooth boundary. 
Now recall that the characteristic functions of smooth sets are in $B V\left(\mathbb{R}^{n}\right)$. (To be more precise, smooth sets $M$ are those for which $\partial M$ is a smooth manifold of codimension 1 and $M$ lies only on one side of the boundary (see [Ta3, p. 84]).)

This means that an analogue of the norm equality in Sobolev spaces (5.9) cannot hold for $B V$-functions.

Lemma 5.4. Let $u \in B V\left(\mathbb{R}^{n}\right) \cap L_{+}^{1}\left(\mathbb{R}^{n}\right)$ and let $u_{H}$ be some polarization of $u$. Then $u_{H} \in B V\left(\mathbb{R}^{n}\right)$ and

$$
\left\|D u_{H}\right\|_{B V} \leq\|D u\|_{B V}
$$

Proof. We choose a sequence of functions $u_{m} \in W_{+}^{1,1}\left(\mathbb{R}^{n}\right)$ which converges to $u$ in $B V\left(\mathbb{R}^{n}\right)$. By Lemma 5.3 the functions $\left(u_{m}\right)_{H}$ are equibounded in $W^{1,1}\left(\mathbb{R}^{n}\right)$. Therefore there are some function $v \in B V\left(\mathbb{R}^{n}\right)$ and a subsequence $u_{m^{\prime}}$ such that

$$
\left(u_{m^{\prime}}\right)_{H} \rightarrow v \quad \text { weakly in } B V\left(\mathbb{R}^{n}\right) \text {. }
$$

On the other hand from the inequalities

$$
\left\|\left(u_{m}\right)_{H}-u_{H}\right\|_{1} \leq\left\|u_{m}-u\right\|_{1}
$$

we conclude that

$$
\left(u_{m}\right)_{H} \longrightarrow u_{H} \quad \text { in } \quad L^{1}\left(\mathbb{R}^{n}\right) .
$$

Now let $\mu_{i}$ denote the Radon-measure which is associated with the weak partial derivative $v_{x_{i}}(i=1, \ldots, n)$. Then we have for every $\varphi \in C_{0}^{\infty}\left(\mathbb{R}^{n}\right)$

$$
\begin{aligned}
\int_{\mathbb{R}^{n}} \varphi d \mu_{i} & \longleftarrow \int_{\mathbb{R}^{n}} \varphi \frac{\partial\left(u_{m^{\prime}}\right)_{H}}{\partial x_{i}} d x \\
& =-\int_{\mathbb{R}^{n}} \varphi_{x_{i}}\left(u_{m^{\prime}}\right)_{H} d x \longrightarrow-\int_{\mathbb{R}^{n}} \varphi_{x_{i}} u_{H} d x,
\end{aligned}
$$

which means that $v=u_{H}$.

Finally the weak lower semi-continuity of the norm gives

$$
\left\|D u_{H}\right\|_{B V} \leq \liminf \left\|\nabla\left(u_{m}\right)_{H}\right\|_{1}=\lim \left\|\nabla u_{m}\right\|_{1}=\|D u\|_{B V} .
$$

Corollary 5.3. The perimeter (in the sense of De Giorgi) of a Caccioppoli set in $\mathbb{R}^{n}$ decreases under polarization, i.e.:

$$
\left\|D \chi\left(E_{H}\right)\right\|_{B V} \leq\|D \chi(E)\|_{B V} \quad \forall \text { Caccioppoli sets } E \subset \mathbb{R}^{n} .
$$

\section{Approximation of symmetrization of Functions}

We show here that every $(k, n)$-Steiner symmetrization, $1 \leq k \leq n$, can be approximated in $L^{p}\left(\mathbb{R}^{n}\right), 1 \leq p<+\infty$, and in $C\left(\mathbb{R}^{n}\right)$ by a sequence of polarizations. Let us mention that any $k$-dimensional Steiner symmetrization of sets can be approximated by a sequence of $(k-1)$-dimensional ones (see Sa for compacts and BLL for measurable sets).

A central role in the approach is played by the following

Lemma 6.1. Let $u \in L_{+}^{p}\left(\mathbb{R}^{n}\right)(1 \leq p<+\infty)$, let $P_{m}$ be polarizations with corresponding halfspaces $H_{m}$ and $u_{m}=\bigcirc_{i=1}^{m} P_{i} u$, and assume that $0 \in H_{m}, m=$ $1,2, \ldots$ Then the sequence $\left\{u_{m}\right\}$ is conditionally compact in $L^{p}\left(\mathbb{R}^{n}\right)$. 
Proof. 1) Since $\|u\|_{p}=\left\|u_{m}\right\|_{p}, m=1,2, \ldots$, the functions $u_{m}$ are equibounded in $L^{p}\left(\mathbb{R}^{n}\right)$.

2) Let $R>0, m \in \mathbb{N}$. Since $P_{m}\left(B_{R}\right)=B_{R}$, we obtain by (3.10)

$$
\int_{B_{R}}\left|u_{m}\right|^{p} d x \leq \int_{B_{R}}\left|P_{m+1}\left(u_{m}\right)\right|^{p}(x) d x=\int_{B_{R}}\left|u_{m+1}\right|^{p} d x .
$$

This means that

$$
\lim _{R \rightarrow+\infty} \int_{\mathbb{R}^{n} \backslash B_{R}}\left|u_{m}\right|^{p} d x=0 \quad \text { uniformly in } m \in \mathbb{N} .
$$

3) Let $v \in C_{0+}^{0,1}\left(\mathbb{R}^{n}\right), h \in \mathbb{R}^{n}$. If $v_{H}$ is any polarization of $v$, we infer from Lemma 5.3 that $v_{H} \in C_{0+}^{0,1}\left(\mathbb{R}^{n}\right)$ and

$$
\left\|v_{H}(\cdot+h)-v_{H}(\cdot)\right\|_{p} \leq|h|\left\|\nabla v_{H}\right\|_{p}=|h|\|\nabla v\|_{p} .
$$

Now for a given $\varepsilon>0$ we choose $v \in C_{0+}^{0,1}\left(\mathbb{R}^{n}\right)$ with

$$
\|u-v\|_{p}<\frac{\varepsilon}{3}
$$

and then $h \in \mathbb{R}^{n}$ with

$$
|h|<\frac{\varepsilon}{3\|\nabla v\|_{p}} .
$$

Setting $v_{m}:=\bigcirc_{i=1}^{m} P_{m}(v), m=1,2, \ldots$, and then applying (6.2) and (3.9) inductively, we conclude

$$
\begin{aligned}
\left\|u_{m}(\cdot+h)-u_{m}(\cdot)\right\|_{p} & \leq\left\|v_{m}(\cdot+h)-v_{m}(\cdot)\right\|_{p}+2\left\|u_{m}-v_{m}\right\|_{p} \\
& \leq\|\nabla v\|_{p}|h|+2\|u-v\|_{p}<\varepsilon
\end{aligned}
$$

This means that

$$
\lim _{|h| \rightarrow 0}\left\|u_{m}(\cdot+h)-u_{m}(\cdot)\right\|_{p}=0 \quad \text { uniformly in } m \in \mathbb{N} .
$$

Now the assertion follows from 1),(6.1),(6.3) and a well-known compactnesscriterion in $L^{p}\left(\mathbb{R}^{n}\right)$ (see [DS, Theorem 8.21]).

A compactness result analogous to Lemma 6.1 holds in the space of continuous functions. A similar observation was made by Baernstein and Taylor in a proof of a convolution-type inequality for a spherical symmetrization [BT, p.252 ff.] and variants of it appeared in some other papers (see e.g. [Be], [Bae2]).

Lemma 6.2. Let $u \in C\left(\mathbb{R}^{n}\right) \cap \mathcal{S}_{+}$, and let $\left\{u_{m}\right\}$ be a sequence of polarizations of $u$ as in Lemma 6.1. Then there are a subsequence $\left\{u_{m^{\prime}}\right\}$ and a function $v \in$ $C\left(\mathbb{R}^{n}\right) \cap \mathcal{S}_{+}$such that

$$
u_{m^{\prime}} \longrightarrow v \quad \text { in } C\left(\mathbb{R}^{n}\right)
$$

and

$$
\omega_{v} \leq \omega_{u} .
$$

Proof. Since $\|u\|_{\infty}=\left\|u_{m}\right\|_{\infty}$, the functions $u_{m}$ are equibounded. From Lemma 4.1 we see that $\omega_{u_{m}} \leq \omega_{u}$, that is, the functions $u_{m}$ are also equicontinuous. The assertion then follows by Arzela's Theorem. 
From now on until the end of section 7 let * denote any $(k, n)$-Steiner-symmetrization $(1 \leq k \leq n)$. Let $\Sigma$ be the symmetry plane of "*", let $\mathcal{H}$ denote the set of all halfspaces $H$ for which the normal to $\partial H$ is orthogonal to $\Sigma$ and let $\mathcal{H}_{0}$ be the set of all halfspaces in $\mathcal{H}$ containing $\Sigma$. Sometimes we will choose a coordinate system $\mathbb{R}^{n} \ni x=(x, y), y \in \mathbb{R}^{k}$, in which $\Sigma$ takes the form $\{y=0\}$.

The next lemma shows how one can identify symmetric situations with the aid of polarizations. Equations (6.6)-(6.9) below follow easily from the definition of polarization and from the "radial symmetry" of symmetrized sets and functions in slices (see property (4.4)).

Lemma 6.3. Let $M \in \mathcal{M}$ and $u \in \mathcal{S}$. Then

$$
\begin{aligned}
& M^{*}=\left(M_{H}\right)^{*} \quad \text { and } \quad u^{*}=\left(u_{H}\right)^{*} \quad \forall H \in \mathcal{H}, \\
& M^{*}=\left(M^{*}\right)_{H} \quad \text { and } \quad u^{*}=\left(u^{*}\right)_{H} \quad \forall H \in \mathcal{H}_{0} \text {, }
\end{aligned}
$$

and

$$
\begin{aligned}
M & =M^{*} & \Longleftrightarrow \quad M & =M_{H} & \forall H \in \mathcal{H}_{0} & \text { and } \\
u & =u^{*} & \Longleftrightarrow \quad u & =u_{H} & \forall H \in \mathcal{H}_{0} . &
\end{aligned}
$$

Furthermore, if $\tilde{M}, \tilde{u}$ denote any translates of $M$, respectively $u$, in a direction orthogonal to $\Sigma$, then

$$
\begin{aligned}
& \tilde{M}=M^{*} \Longleftrightarrow M=M_{H} \quad \text { or } \quad \sigma_{H}(M)=M_{H} \quad \forall H \in \mathcal{H} \quad \text { and } \\
& \tilde{u}=u^{*} \Longleftrightarrow u=u_{H} \quad \text { or } \quad \sigma_{H}(u)=u_{H} \quad \forall H \in \mathcal{H} .
\end{aligned}
$$

The next lemma is crucial for the approximation of symmetrized functions.

Lemma 6.4. Let $u \in L_{+}^{p}\left(\mathbb{R}^{n}\right)(1 \leq p<+\infty)$, and assume that $u \neq u^{*}$. Then there is some halfspace $H \in \mathcal{H}_{0}$ such that

$$
\left\|u_{H}-u^{*}\right\|_{p}<\left\|u-u^{*}\right\|_{p}
$$

Proof. We follow the ideas of [BT, p.252 ff.].

Let $H \in \mathcal{H}_{0}$. Then by $(6.7)$ we have $\left(u^{*}\right)_{H}=u^{*}$, and by a partition into cases we can verify that

$$
\left|u_{H}(x)-u^{*}(x)\right|^{p}+\left|u_{H}(\bar{x})-u^{*}(\bar{x})\right|^{p} \leq\left|u(x)-u^{*}(x)\right|^{p}+\left|u(\bar{x})-u^{*}(\bar{x})\right|^{p}
$$

$$
\forall x \in H \text {. }
$$

An integration of (6.11) over $H$ yields

$$
\left\|u_{H}-u^{*}\right\|_{p} \leq\left\|u-u^{*}\right\|_{p} .
$$

Therefore to prove (6.10) it suffices to show that for a suitable choice of $H \in \mathcal{H}_{0}$ the inequality (6.11) becomes strict on a subset of $H$ of positive measure.

Since $u \neq u^{*}$, we find some number $c>0$ such that

$$
L^{n}\left(\{u>c\} \Delta\left\{u^{*}>c\right\}\right)>0 .
$$

By the equimeasurability we have $L^{n}(\{u>c\})=L^{n}\left(\left\{u^{*}>c\right\}\right)$. We consider two cases

(i) $k=n$.

Let $x^{1}$ and $x^{2}$ be density points of the sets $\{u>c\} \backslash\left\{u^{*}>c\right\}$ and $\left\{u^{*}>c\right\} \backslash\{u>$ $c\}$, respectively. Then we can choose a halfspace $H$ such that $x^{1}=\overline{x^{2}}$ and $x^{2} \in H$. 
(Note that from $u^{*}\left(x^{1}\right) \leq c<u^{*}\left(x^{2}\right)$ it follows that $0 \in H$ and thus $H \in \mathcal{H}_{0}$ !) Hence there is a subset $N$ of $H$ of positive measure (which contains $x^{2}$ !) such that

$$
u^{*}(x)>c \geq u(x), \quad u^{*}(\bar{x}) \leq c<u(\bar{x}) \quad \forall x \in N .
$$

But this means that the inequality $(6.11)$ becomes strict on the set $N$, q.e.d.

(ii) $1 \leq k \leq n-1$.

Setting

$$
\left.M:=\left\{x^{\prime} \in \mathbb{R}^{n-k}: L^{k}\left(\left\{u\left(x^{\prime}, \cdot\right)>c\right\} \Delta\left\{u^{*}\left(x^{\prime}, \cdot\right)>c\right)\right\}\right)>0\right\},
$$

we see that $L^{n-k}(M)>0$. Let $x_{0}^{\prime}$ be a density point of $M$. Then

$$
L^{k}\left(\left\{u\left(x_{0}^{\prime}, \cdot\right)>c\right\} \backslash\left\{u^{*}\left(x_{0}^{\prime}, \cdot\right)>c\right\}\right)=L^{k}\left(\left\{u^{*}\left(x_{0}^{\prime}, \cdot\right)>c\right\} \backslash\left\{u\left(x_{0}^{\prime}, \cdot\right)>c\right\}\right)>0 .
$$

On the other hand, let $y_{1}$ and $y_{2}$ be density points of the sets $\left\{u\left(x_{0}^{\prime}, \cdot\right)>c\right\} \backslash$ $\left\{u^{*}\left(x_{0}^{\prime}, \cdot\right)>c\right\}$ and $\left\{u^{*}\left(x_{0}^{\prime}, \cdot\right)>c\right\} \backslash\left\{u\left(x_{0}^{\prime}, \cdot\right)>c\right\}$, respectively. There is exactly one halfspace $H$ for which $\left(x_{0}^{\prime}, y_{2}\right) \in H$ and $\overline{\left(x_{0}^{\prime}, y_{1}\right)}=\left(x_{0}^{\prime}, y_{2}\right)$. Then clearly $H \in \mathcal{H}_{0}$, and there is again some subset $N$ of $H$ with positive measure (containing $\left.\left(x_{0}^{\prime}, y_{2}\right) !\right)$ such that the relations (6.12) are satisfied, and we conclude as before.

Theorem 6.1. Let $u \in L_{+}^{p}\left(\mathbb{R}^{n}\right)(1 \leq p<+\infty)$, let $P_{m}$ be polarizations with corresponding halfspaces $H_{m} \in \mathcal{H}_{0}$ and let $u_{m}:=\bigcirc_{i=1}^{m} P_{i} u, m=1,2, \ldots$. Further let the $H_{m}$ 's be chosen in such a way that

$$
\begin{aligned}
&\left\|u_{m+1}-u^{*}\right\|_{p}=\min \left\{\left\|\left(u_{m}\right)_{H}-u^{*}\right\|_{p}: H \in \mathcal{H}_{0}\right\}, \\
& m=0,1, \ldots, \quad u_{0}:=u .
\end{aligned}
$$

Then

$$
u_{m} \longrightarrow u^{*} \quad \text { in } L^{p}\left(\mathbb{R}^{n}\right) .
$$

Proof. First observe that in view of Lemma 5.2 the minimum in (6.13) is indeed attained for some halfspace $H_{m+1} \in \mathcal{H}_{0}$. Then by Lemma 6.1 there are some function $v \in L_{+}^{p}\left(\mathbb{R}^{n}\right)$ and a subsequence $u_{m^{\prime}}$ such that:

$$
u_{m^{\prime}} \longrightarrow v \text { in } L^{p}\left(\mathbb{R}^{n}\right),
$$

and in view of Lemma 3.1 we have $v^{*}=u^{*}$. Now assume that $v \neq u^{*}$. By Lemma 6.4 we can choose a hyperplane such that

$$
\left\|v_{H}-u^{*}\right\|_{p}<\left\|v-u^{*}\right\|_{p}
$$

It follows that

$$
\begin{aligned}
& \left\|\left(u_{m^{\prime}}\right)_{H}-u^{*}\right\|_{p}-\left\|u_{m^{\prime}}-u^{*}\right\|_{p} \\
& \quad \leq\left\|\left(u_{m^{\prime}}\right)_{H}-v_{H}\right\|_{p}+\left\|v_{H}-u^{*}\right\|_{p}+\left\|u_{m^{\prime}}-v\right\|_{p}-\left\|v-u^{*}\right\|_{p} \\
& \quad \leq 2\left\|u_{m^{\prime}}-v\right\|_{p}+\left\|v_{H}-u^{*}\right\|_{p}-\left\|v-u^{*}\right\|_{p} \\
& \quad \longrightarrow\left\|v_{H}-u^{*}\right\|_{p}-\left\|v-u^{*}\right\|_{p}<0 \quad \text { as } m^{\prime} \rightarrow+\infty .
\end{aligned}
$$

On the other hand the sequence $\left\|u_{m}-u^{*}\right\|_{p}$ is monotonically decreasing; hence

$$
\left\|u_{m+1}-u^{*}\right\|_{p}-\left\|u_{m}-u^{*}\right\|_{p} \longrightarrow 0 \quad \text { as } m \rightarrow+\infty .
$$

Together with (6.15) this contradicts the minimality property (6.13). 
Theorem 6.2. Let $u \in C\left(\mathbb{R}^{n}\right) \cap L_{+}^{1}\left(\mathbb{R}^{n}\right)$ and let the functions $u_{m}$ be defined as in Lemma 6.5, whereby the condition (6.13) is satisfied with $p=1$. Then

$$
u_{m} \longrightarrow u^{*} \quad \text { in } C\left(\mathbb{R}^{n}\right) .
$$

Proof. By the previous lemma we have

$$
u_{m} \longrightarrow u^{*} \quad \text { in } L^{1}\left(\mathbb{R}^{n}\right)
$$

and the functions $u_{m}$ are equicontinuous in view of Lemma 4.1. Because of Lemma 6.2 we have also

$$
u_{m^{\prime}} \longrightarrow v \quad \text { in } C\left(\mathbb{R}^{n}\right)
$$

for a subsequence $u_{m^{\prime}}$ and $\omega_{v} \leq \omega_{u}$. Thus $v=u^{*}$ and the assertion follows.

Corollary 6.1. Let $u \in C\left(\mathbb{R}^{n}\right) \cap \mathcal{S}_{+}$. Then

$$
\omega_{u^{*}} \leq \omega_{u} \text {. }
$$

Proof. If $u \in L^{1}\left(\mathbb{R}^{n}\right)$, then (6.17) follows by the above proof. In the general case we choose a sequence $u_{m}$ of functions in $C\left(\mathbb{R}^{n}\right) \cap L_{+}^{1}\left(\mathbb{R}^{n}\right)$ converging to $u$ in $C\left(\mathbb{R}^{n}\right)$. Then by (3.9) we have

$$
\left\|\left(u_{m}\right)^{*}-u^{*}\right\|_{\infty} \leq\left\|u_{m}-u\right\|_{\infty}, \quad m=1,2, \ldots,
$$

and the assertion follows.

Remark 6.1. Lemma 3.7 and Corollary 6.1 show that all $(k, n)$-Steiner symmetrizations, $1 \leq k \leq n$, are smoothing. By Lemma 3.3 and 3.4 this means that they are also open and compact.

As a byproduct we get a special case of the well-known Brunn-Minkowski inequality in $\mathbb{R}^{n}$ (see e.g. [Ha, p.174 ff.]). We will not discuss the equality sign in (6.18) below, a question which was completely solved in various ways (see e.g. [Ha], [BuZ] and [Sch] $)$.

Theorem 6.3. Let $F \in \mathcal{F}$ and $r>0$. Then

$$
\mathcal{L}^{n}\left(F_{r}\right) \geq \mathcal{L}^{n}\left(\left(F^{\star}\right)_{r}\right) .
$$

\section{Approximation of Symmetrization of SETS}

The comparison theorems for symmetrizations from section 10 need some stronger convergence results than Lemmata 6.5, 6.6 for domains. Therefore we shall investigate the convergence of sequences of polarizations of open and compact sets in the Hausdorff metric. (Note that in fact we will exploit later only the second assertion (7.2) of Lemma 7.1 below.)

Lemma 7.1. Let $G, G^{\prime} \in \mathcal{G}_{b}$ with $G^{\prime} \Subset G$. Then there exist polarizations $P_{i}$ with corresponding halfspaces $H_{i} \in \mathcal{H}_{0}, i=1, \ldots, m_{0}$, such that

$$
\begin{aligned}
& \bigcirc_{i=1}^{m_{0}} P_{i}(G) \ni\left(G^{\prime}\right)^{*} \quad \text { and } \\
& \bigcirc_{i=1}^{m_{0}} P_{i}\left(G^{\prime}\right) \Subset G^{*} .
\end{aligned}
$$

Proof. We choose a function $u \in C\left(\mathbb{R}^{n}\right) \cap \mathcal{S}_{+}$satisfying

$$
\text { supp } u \subset G, \quad 0 \leq u \leq 1 \text { in } G \backslash \overline{G^{\prime}} \text { and } u>1 \text { in } G^{\prime} .
$$

By Lemma 6.6 there are polarizations $P_{i}$ with corresponding halfspaces $H_{i} \in$ $\mathcal{H}_{0}, i=1,2, \ldots$, such that

$$
\bigcirc_{i=1}^{m} P_{i} u=: u_{m} \longrightarrow u^{*} \text { in } C\left(\mathbb{R}^{n}\right) .
$$


By setting $G^{m}:=\bigcirc_{i=1}^{m} P_{i}(G)$ and $\left(G^{\prime}\right)^{m}:=\bigcirc_{i=1}^{m} P_{i}\left(G^{\prime}\right), m=1,2, \ldots$, we derive from (7.3) that

$$
\text { supp } u^{*} \subset G^{*}, \quad 0 \leq u^{*} \leq 1 \quad \text { in } G^{*} \backslash \overline{\frac{\left(G^{\prime}\right)^{*}}{\left(G^{\prime}\right)^{m}}} \quad u^{*}>1 \text { in }\left(G^{\prime}\right)^{*} \quad \text { and }
$$
supp $u_{m} \subset G^{m}, \quad 0 \leq u_{m} \leq 1 \quad$ in $G^{m} \backslash \overline{\left(G^{\prime}\right)^{m}}$,

$$
u_{m}>1 \text { in }\left(G^{\prime}\right)^{m}, m=1,2, \ldots
$$

We infer from (7.4) and (7.5) that for sufficiently large $m$ (say $m \geq m_{0}$ )

$$
u_{m}>(1 / 2) \text { in }\left(G^{\prime}\right)^{*} \text { and } u_{m}<(1 / 2) \text { in } \mathbb{R}^{n} \backslash G^{*},
$$

which implies that

$$
G^{m} \supset \operatorname{supp} u_{m} \supset\left(G^{\prime}\right)^{*} \text { and }\left(G^{\prime}\right)^{m} \subset \overline{\left(G^{\prime}\right)^{m}} \subset G^{*} \quad \forall m \geq m_{0} .
$$

Lemma 7.2. Let $F \in \mathcal{F}$. Then there are polarizations $P_{i}$ with corresponding halfspaces $H_{i} \in \mathcal{H}_{0}, i=1,2, \ldots$, such that

$$
\lim _{m \rightarrow \infty} d\left(\bigcirc_{i=1}^{m} P_{i}(F), F^{*}\right)=0 .
$$

Proof. We introduce a function $u$ by

$$
u(x):= \begin{cases}1-\operatorname{dist}\{x ; F\} & \text { if } x \in\left(F+\overline{B_{1}}\right), \\ 0 & \text { if } x \in \mathbb{R}^{n} \backslash\left(F+\overline{B_{1}}\right) .\end{cases}
$$

By Lemma 6.6 there are polarizations $P_{i}$ with corresponding halfspaces $H_{i} \in$ $\mathcal{H}_{0}, i=1,2, \ldots$, such that

$$
\bigcirc_{i=1}^{m} P_{i} u=: u_{m} \longrightarrow u^{*} \quad \text { in } C\left(\mathbb{R}^{n}\right) .
$$

We set $F^{m}:=\bigcirc_{i=1}^{m} P_{i}(F), m=1,2, \ldots$ Then (7.6) can equivalently be written as

$$
\begin{array}{lll}
\inf \left\{r>0: F^{m} \subset\left(F^{*}\right)_{r}\right\} \longrightarrow 0 & \text { and } & \\
\inf \left\{r>0: F^{*} \subset\left(F^{m}\right)_{r}\right\} \longrightarrow 0 & \text { as } \quad m \rightarrow \infty
\end{array}
$$

It remains to prove (7.8) and (7.9).

1) First assume that (7.8) is not true. Then there are some $r \in(0,1)$ and points $x_{m} \in F^{m} \backslash\left(F^{*}\right)_{r}, m=1,2, \ldots$. We can assume that $x_{m} \rightarrow x$ and $x \notin\left(F^{*}\right)_{(r / 2)}$. Since $u_{m}=1$ in $F^{m}, m=1,2, \ldots$, and $u^{*}<1-\varepsilon$ in $\mathbb{R}^{n} \backslash\left(F^{*}\right)_{(r / 2)}$ for some $\varepsilon>0$, we conclude that

$$
\left|u_{m}\left(x_{m}\right)-u^{*}\left(x_{m}\right)\right|>(\varepsilon / 2) \quad \forall \text { large enough } m,
$$

which contradicts (7.7).

2) Next assume that (7.9) is not true. Then there are some $r \in(0,1)$ and points $x_{m} \in F^{*} \backslash\left(F^{m}\right)_{r}, m=1,2, \ldots$ We can assume that $x_{m} \rightarrow x_{0}=\left(x_{0}^{\prime}, y_{0}\right) \in F^{*}$. If $m$ is large enough (say $m \geq m_{0}$ ), we have

$$
B_{r / 2}\left(x_{0}\right) \cap F^{m}=\emptyset \text {. }
$$

Now we study the situation on the hyperplane $\left\{x^{\prime}=x_{0}^{\prime}\right\}$. Recall that

$$
\begin{aligned}
& \mathcal{L}^{k}\left(F\left(x_{0}^{\prime}\right)\right)=\mathcal{L}^{k}\left(F^{*}\left(x_{0}^{\prime}\right)\right)=\mathcal{L}^{k}\left(F^{m}\left(x_{0}^{\prime}\right)\right), m=1,2, \ldots, \quad \text { and } \\
& F^{*}\left(x_{0}^{\prime}\right) \text { is a ball in } \mathbb{R}^{k} \text { which is centered in the origin. }
\end{aligned}
$$

Two cases (i) and (ii) are possible. 
(i) $\mathcal{L}^{k}\left(F^{*}\left(x_{0}^{\prime}\right)\right)=0$. Then $F^{*}\left(x_{0}^{\prime}\right)=\{0\}$ by (7.11), and in view of (7.10) there are points $\tilde{y}_{m} \in F^{m}\left(x_{0}^{\prime}\right)$ with $\left|\tilde{y}_{m}\right|>r / 2$.

(ii) $\mathcal{L}^{k}\left(F^{*}\left(x_{0}^{\prime}\right)\right)>0$. Then $\mathcal{L}^{k}\left(\left\{y \in \mathbb{R}^{k}:\left|y-y_{0}\right|<r / 2\right\} \cap F^{*}\left(x_{0}^{\prime}\right)\right)>0$. By (7.10) and (7.11) this means that there are some number $\delta_{1}>0$ and points $\tilde{y}_{m} \in F^{m}\left(x_{0}^{\prime}\right)$ with dist $\left\{\tilde{y}_{m} ; F^{*}\left(x_{0}^{\prime}\right)\right\} \geq \delta_{1}$.

Thus, by setting $\delta_{2}:=\min \left\{r / 2 ; \delta_{1}\right\}$, in both cases (i) and (ii) we find points $\tilde{y}_{m} \in F^{m}\left(x_{0}^{\prime}\right)$ with

$$
\operatorname{dist}\left\{\tilde{y}_{m} ; F^{*}\left(x_{0}^{\prime}\right)\right\} \geq \delta_{2}
$$

In view of (7.11) $F^{*}$ has a representation

$$
F^{*}=\left\{\left(x^{\prime}, y\right):|y| \leq \mathcal{L}^{k}\left(F\left(x^{\prime}\right)\right), x^{\prime} \in F^{\prime}\right\},
$$

where $F^{\prime}$ is some compact in $\mathbb{R}^{n-k}$ and $\mathcal{L}^{k}\left(F\left(x^{\prime}\right)\right)$ is upper semicontinuous. Hence by setting $\tilde{x}_{m}:=\left(x_{0}^{\prime}, \tilde{y}_{m}\right)$, we see from (7.12) that there is some number $\delta>0$ such that

$$
\operatorname{dist}\left\{\tilde{x}_{m} ; F^{*}\right\}>\delta \quad \forall m \geq m_{0} .
$$

Thus we have $u_{m}\left(\tilde{x}_{m}\right)=1$ and $u^{*}\left(\tilde{x}_{m}\right)<1-\tilde{\varepsilon}$ for some $\tilde{\varepsilon}>0$. By arguing similarly as in part 1) we obtain a contradiction to (7.7). The lemma is proved.

\section{INTEGRAL INEQUALITIES FOR SYMMETRIZATIONS}

Using the approach of section 5 we can derive many well-known integral inequalities in the theory of symmetrizations.

The idea is the same in all cases: First one proves an analogous inequality where the symmetrizations of functions are replaced by some polarizations. In most cases this proof will be much simpler. (Sometimes the integral inequality can be further reduced to a pointwise one for the integrands (see e.g. Lemma 8.1 below).)

After that one approximates the symmetrized function by sequences of polarizations. Together with some convergence properties of the integrals this leads to the final inequality.

To illustrate the method, we state now two well-known convolution-type inequalities in $\mathbb{R}^{n}$ (see [Be, p.4818] and [Bae2] Corollary 2]). Note that these inequalities are proved for the special case $j(z)=z^{2}$ in [BT, Lemma 1], and they hold true also for the sphere $S^{n}$ and the hyperbolic space $\mathbb{H}^{n}$. We present them in a slightly generalized form.

Lemma 8.1. Let $H \in \mathcal{H}$, let $u, v, w \in \mathcal{S}_{+}$with $w=w^{*}$ and let $j$ be a Young function. Then

$$
\iint_{\mathbb{R}^{2 n}} j(|u(x)-v(y)|) w(x-y) d x d y \geq \iint_{\mathbb{R}^{2 n}} j\left(\left|u_{H}(x)-v_{H}(y)\right|\right) w(x-y) d x d y,
$$

if the left-hand side in (8.1) converges.

Proof. We use the notations of section 5. Let $x, y \in H$. Since $j$ is convex, it follows from elementary analysis that

$$
j(|u(\bar{x})-v(y)|)+j(|u(x)-v(\bar{y})|) \geq j\left(\left|u_{H}(\bar{x})-v_{H}(y)\right|\right)+j\left(\left|u_{H}(x)-v_{H}(\bar{y})\right|\right) .
$$


In view of $w=w^{*}$ and the assumption on $H$ we have

$$
w(x-y)=w(\bar{x}-\bar{y}) \geq w(\bar{x}-y)=w(x-\bar{y}) .
$$

Together with (8.2) this leads to the inequality

$$
\begin{aligned}
j(\mid u(x)- & v(y) \mid) w(x-y)+j(|u(\bar{x})-v(y)|) w(\bar{x}-y) \\
& +j(|u(x)-v(\bar{y})|) w(x-\bar{y})+j(|u(\bar{x})-v(\bar{y})|) w(\bar{x}-\bar{y}) \\
\geq & j\left(\left|u_{H}(x)-v_{H}(y)\right|\right) w(x-y)+j\left(\left|u_{H}(\bar{x})-v_{H}(y)\right|\right) w(\bar{x}-y) \\
& +j\left(\left|u_{H}(x)-v_{H}(\bar{y})\right|\right) w(x-\bar{y})+j\left(\left|u_{H}(\bar{x})-v_{H}(\bar{y})\right|\right) w(\bar{x}-\bar{y}) .
\end{aligned}
$$

Then an integration over $H \times H$ yields (8.1).

Lemma 8.2. Let $u, v, w \in \mathcal{S}_{+}$with $w=w^{*}$ and let $j$ be a Young-function. Then

$$
\iint_{\mathbb{R}^{2 n}} j(|u(x)-v(y)|) w(x-y) d x d y \geq \iint_{\mathbb{R}^{2 n}} j\left(\left|u^{*}(x)-v^{*}(y)\right|\right) w(x-y) d x d y,
$$

provided the left-hand side in (8.3) converges.

Proof. First observe that in view of the nonexpansivity (3.7), we can restrict ourselves to the case that $u$ and $v$ are continuous functions with bounded support. Then we define inductively two sequences $u_{m}$ and $v_{m}$ of polarizations of $u$ and $v$, as in Theorem 6.1, where the corresponding halfspaces $H_{m}$ are chosen in such a way that the minimality property (6.13) is satisfied. By Theorem 6.2 the sequences $u_{m}$ and $v_{m}$ converge in $C\left(\mathbb{R}^{n}\right)$ to $u^{*}$ and $v^{*}$, respectively. Then (8.3) follows by applying Lemma 8.1 inductively.

Remark 8.1. (8.3) includes a special case of Riesz inequality (see [Bae2]).

In fact, let $j(z)=z^{2}$ and let $u, v, w$ as in Lemma 8.2. Then

$$
\iint_{\mathbb{R}^{n}} u(x) v(y) w(x-y) d x d y \leq \iint_{\mathbb{R}^{n}} u^{*}(x) v^{*}(y) w(x-y) d x d y,
$$

if the left-hand side in (8.4) converges.

(Note that, in contrast to the general version of (8.4) (see e.g. [Ka1, p.25]), the third function $w$ in (8.4) is already symmetrized.)

Recently one of the authors (see $[\mathrm{Br}]$ ) used polarization to show the symmetry of local minimizers of some variational problems with potentials. Those problems may describe equilibrium states in continuum mechanics, e.g. of plasma regions, rotating stars and liquids (see [F Chapter 4]). The idea consists in combining the continuity property of the polarization (Lemma 5.2), the "identification" lemma 6.3 and the convolution inequality for the polarization (Lemma 8.1). Theorem 8.1 below generalizes a corresponding result of $[\mathrm{Br}]$ to the case of $(k, n)$-Steiner symmetrization, $1 \leq k \leq n$. The proof from $[\mathrm{Br}]$ carries over without difficulties to the general case.

Theorem 8.1. Let $K$ be a closed subset of $L_{+}^{p}\left(\mathbb{R}^{n}\right), 1 \leq p<+\infty$, and let $K$ have the property that if $v \in K$, then also $v^{*} \in K$. Furthermore, let $\varphi, \psi$ be real 
continuous functions on $\mathbb{R}_{0}^{+}$, let $j$ be a Young function, $g, w \in \mathcal{S}_{+}$with $g=g^{*}$ and $w=w^{*}$, and let $J$ be defined by

$$
\begin{aligned}
J(v):= & \iint_{\mathbb{R}^{2 n}} j(|v(x)-v(y)|) w(x-y) d x d y-\int_{\mathbb{R}^{n}} \varphi(v(x)) g(x) d x \\
& +\int_{\mathbb{R}^{n}} \psi(v(x)) d x, \quad v \in K .
\end{aligned}
$$

Finally suppose that the functions $g, w, j$ satisfy one of the following conditions:

(i) The function $\tilde{g}$, defined by

$$
\tilde{g}(x, r):=g(x, y) \quad(r=|y|)
$$

is strictly decreasing in $r$.

(ii) The function $\tilde{w}$, defined by

$$
\tilde{w}(x, r):=w(x, y) \quad(r=|y|)
$$

is strictly decreasing in $r$, and $j$ is strictly convex.

Then, if $u$ is a local minimum of $J$ in $K$, we have $u^{*}(x)=u(\sigma x)$, where $\sigma$ is some translation in a direction orthogonal to $\Sigma$.

Next we give elementary proofs of some well-known Dirichlet-type inequalities for functions and their symmetrizations (see e.g. Bae2, Ka1 and the literature cited therein).

Remark 8.2. Let $u \in W_{+}^{1, p}\left(\mathbb{R}^{n}\right)$. Using the limit process of the previous section we cannot hope that the polarizations $u_{m}$ converge strongly to $u^{*}$ in $W^{1, p}\left(\mathbb{R}^{n}\right)$. (Otherwise there would be $\|\nabla u\|_{p}=\left\|\nabla u^{*}\right\|_{p}$, which is generally not true!)

Theorem 8.2. Let $u \in W_{+}^{1, p}\left(\mathbb{R}^{n}\right)(1 \leq p<+\infty)$. Then $u^{*} \in W_{+}^{1, p}\left(\mathbb{R}^{n}\right)$ and

$$
\left\|\nabla u^{*}\right\|_{p} \leq\|\nabla u\|_{p}
$$

Furthermore, if $V$ is some linear subspace which either contains all " $y$-directions" $x_{n-k+1}, \ldots, x_{n}$, or is orthogonal to each of these directions, then

$$
\left\|\nabla_{V} u^{*}\right\|_{p} \leq\left\|\nabla_{V} u\right\|_{p} .
$$

Proof. Let $u_{m}$ be the sequence of polarizations of $u$ defined by Theorem 6.1 , which converges to $u^{*}$ in $L^{p}\left(\mathbb{R}^{n}\right)$. We consider 2 cases.

(i) Let $1<p<+\infty$.

Since

$$
\left\|\nabla u_{m}\right\|_{p}=\|\nabla u\|_{p}
$$

by (5.9) we find a function $v \in W^{1, p}\left(\mathbb{R}^{n}\right)$ and a subsequence $u_{m^{\prime}}$ such that

$$
u_{m^{\prime}} \rightarrow v \quad \text { weakly in } W^{1, p}\left(\mathbb{R}^{n}\right) .
$$

This means that for every $\varphi \in C_{0}^{\infty}\left(\mathbb{R}^{n}\right)$ and $i \in\{1, \ldots, n\}$

$$
\begin{aligned}
\int_{\mathbb{R}^{n}} \varphi v_{x_{i}} d x \longleftarrow & \int_{\mathbb{R}^{n}} \varphi \frac{\partial\left(u_{m^{\prime}}\right)}{\partial x_{i}} d x \\
& =-\int_{\mathbb{R}^{n}} \varphi_{x_{i}} u_{m^{\prime}} d x \longrightarrow-\int_{\mathbb{R}^{n}} \varphi_{x_{i}} u^{*} d x,
\end{aligned}
$$

that is, $v=u^{*}$. In view of the lower semi-continuity of the norm it follows that

$$
\left\|\nabla u^{*}\right\|_{p} \leq \liminf \left\|\nabla\left(u_{m}\right)\right\|_{p}=\|\nabla u\|_{p} .
$$


Using the equations (5.10) one proves (8.7) analogously.

(ii) Let $p=1$.

By Lemma 5.3 the functions $\left|\nabla u_{m}\right|$ and $|\nabla u|$ are rearrangements of each other. This means that for every $\delta>0$

$$
\begin{aligned}
\sup \left\{\int_{E}\left|\left(u_{m}\right)_{x_{i}}\right| d x: L^{n}(E) \leq \delta\right\} & \leq \sup \left\{\int_{E}\left|\nabla u_{m}\right| d x: L^{n}(E) \leq \delta\right\} \\
& =\sup \left\{\int_{E}|\nabla u| d x: L^{n}(E) \leq \delta\right\} .
\end{aligned}
$$

Hence, if $E_{k}$ is any sequence of measurable sets with $\lim L^{n}\left(E_{k}\right)=0$, we infer that

$$
\sup \left\{\int_{E_{k}}\left|\left(u_{m}\right)_{x_{i}}\right| d x: m \in \mathbb{N}\right\} \longrightarrow 0, \quad \text { as } k \rightarrow+\infty \text {. }
$$

Applying a well-known weak compactness-criterion in $L^{1}\left(\mathbb{R}^{n}\right)$ (see [A, p.199]), we again can extract a subsequence $u_{m^{\prime}}$ converging weakly in $W^{1,1}\left(\mathbb{R}^{n}\right)$. Then proceeding as in case (i) the assertion follows in the case $p=1$ too.

Corollary 8.1. Inequalities (8.7) contain the following special cases, $(1 \leq i \leq n)$ :

$$
\left\|\frac{\partial u^{*}}{\partial x_{i}}\right\|_{p} \leq\left\|\frac{\partial u}{\partial x_{i}}\right\|_{p}
$$

and

$$
\left\|\nabla_{y} u^{*}\right\|_{p} \leq\left\|\nabla_{y} u\right\|_{p} .
$$

(Here $\nabla_{y}:=\left(\frac{\partial}{\partial x_{n-k+1}}, \ldots, \frac{\partial}{\partial x_{n}}\right)$ is the gradient with respect to $y$.)

A result analogous to Theorem 8.2 holds for Lipschitz functions

Corollary 8.2. Let $u \in W^{1, \infty}\left(\mathbb{R}^{n}\right) \cap L_{+}^{1}\left(\mathbb{R}^{n}\right)$. Then $u^{*} \in W^{1, \infty}\left(\mathbb{R}^{n}\right) \cap L_{+}^{1}\left(\mathbb{R}^{n}\right)$ and (8.6) holds with $p=\infty$.

Proof. Since $u$ is Lipschitz continuous, we infer from Theorem 6.2 that $u^{*}$ is Lipschitz continuous too. By Rademacher's Theorem this means that $u^{*} \in W^{1, \infty}\left(\mathbb{R}^{n}\right)$, and inequality (8.6) follows from (6.17).

The following corollary can be proved analogously to Corollary 5.1 by replacing the polarization by the symmetrization.

Corollary 8.3. Let $\Omega \subset \mathbb{R}^{n}$ be an open set and $u \in W_{0+}^{1, p}(\Omega)(1 \leq p<+\infty)$. Then $u^{*} \in W_{0+}^{1, p}\left(\Omega^{*}\right)$ and formulas (8.6)-(8.9) hold.

It is also easy to prove an inequality for convex functionals.

Theorem 8.3. Let $u \in W_{+}^{1,1}\left(\mathbb{R}^{n}\right), k \in\{1, \ldots, n\}$, and let $j$ be a Young-function. Then

$$
\int_{\mathbb{R}^{n}} j\left(\left|\nabla u^{*}\right|\right) d x \leq \int_{\mathbb{R}^{n}} j(|\nabla u|) d x,
$$

if the integral on the right-hand side of (8.10) converges.

Furthermore, if $V$ is chosen as in Theorem 8.2, then

$$
\int_{\mathbb{R}^{n}} j\left(\left|\nabla_{V} u^{*}\right|\right) d x \leq \int_{\mathbb{R}^{n}} j\left(\left|\nabla_{V} u\right|\right) d x,
$$

in the case that the integral on the right-hand side of (8.11) converges. 
Proof. Assume first that $u \in C_{0+}^{0,1}\left(\mathbb{R}^{n}\right)$. If we choose a sequence $u_{m}$ of polarizations of $u$ converging to $u^{*}$ in $L^{1}\left(\mathbb{R}^{n}\right)$, we conclude from Lemma 5.3

$$
\int_{\mathbb{R}^{n}} j\left(\left|\nabla u_{m}\right|\right) d x=\int_{\mathbb{R}^{n}} j(|\nabla u|) d x .
$$

Because of the weak lower semi-continuity of the integral functional this leads to

$$
\int_{\mathbb{R}^{n}} j(|\nabla u|) d x=\liminf \int_{\mathbb{R}^{n}} j\left(\left|\nabla u_{m}\right|\right) d x \geq \int_{\mathbb{R}^{n}} j\left(\left|\nabla u^{*}\right|\right) d x .
$$

If $u \in W_{+}^{1,1}\left(\mathbb{R}^{n}\right)$, we choose a sequence $v_{m} \in C_{0+}^{0,1}\left(\mathbb{R}^{n}\right)$ such that

$$
v_{m} \longrightarrow u \quad \text { in } W^{1,1}\left(\mathbb{R}^{n}\right)
$$

and

$$
\int_{\mathbb{R}^{n}} j\left(\left|\nabla v_{m}\right|\right) d x \longrightarrow \int_{\mathbb{R}^{n}} j(|\nabla u|) d x
$$

This means that we have for a subsequence $v_{m^{\prime}}$

$$
\left(v_{m^{\prime}}\right)^{*} \rightarrow u^{*} \quad \text { weakly in } W^{1,1}\left(\mathbb{R}^{n}\right),
$$

and we conclude again by the weak lower semi-continuity of the functionals.

One proves (8.11) analogously.

An analogue of Theorem 8.2 holds for $B V$-functions. Theorem 8.4 below can be proved analogously to Lemma 5.4 by replacing polarization by symmetrization.

Theorem 8.4. Let $u \in B V\left(\mathbb{R}^{n}\right) \cap L_{+}^{1}\left(\mathbb{R}^{n}\right)$. Then $u^{*} \in B V\left(\mathbb{R}^{n}\right) \cap L_{+}^{1}\left(\mathbb{R}^{n}\right)$ and

$$
\left\|D u^{*}\right\|_{B V} \leq\|D u\|_{B V} .
$$

Choosing for $u$ in (8.12) a characteristic function of a set of finite perimeter we derive the well-known isoperimetric inequality. Again, as in the case of Theorem 6.3 , we will not discuss the equality sign in (8.13) below. A complete study of the isoperimetric problem can be found in the survey article [Ta3]. (For further sources see [BuZ], [Ha] and $[\mathrm{Sch}$. .)

Theorem 8.5 (Isoperimetric inequality in $\mathbb{R}^{n}$ ). Let $E$ be a Caccioppoli set in $\mathbb{R}^{n}$. Then

$$
\left\|D \chi\left(E^{*}\right)\right\|_{B V} \leq\|D \chi(E)\|_{B V} .
$$

\section{TWO-POINT COMPARISON RESULTS FOR THE POLARIZATION}

In this section some boundary and initial value problems are compared with similar problems in which the domain and the data are replaced by polarized ones. Using no more than the maximum principle we obtain some pointwise inequalities for the solutions. This method was applied in $[\mathrm{So} 2]$ to some problems on harmonic measures, Green's functions and the Poincaré metric.

By exploiting several approximation arguments, we will obtain from these comparison theorems some well-known analogues for the $(k, n)$-Steiner symmetrization (see section 10). Those problems were extensively studied during the last decade (see [ALT1], [ALT2] Bae2] and the cited literature therein). In a forthcoming paper [BS] we will prove comparison theorems for some type of continuous $(k, n)$-Steiner symmetrization in a similar way. 
It is useful to work with the following partial ordering relation. Let $H$ be some halfspace in $\mathbb{R}^{n}$. If $f, g \in L_{+}^{1}\left(\mathbb{R}^{n}\right)$, we write

$$
f \prec_{H} g \Longleftrightarrow \int_{\mathbb{R}^{n}} f h d x \leq \int_{\mathbb{R}^{n}} g_{H} h_{H} d x \quad \forall h \in L_{+}^{\infty}\left(\mathbb{R}^{n}\right) .
$$

Lemma 9.1. Let $H$ be some halfspace in $\mathbb{R}^{n}$ and $f, g \in L_{+}^{1}\left(\mathbb{R}^{n}\right)$. Then

$$
\begin{aligned}
& f \prec_{H} g \\
& \Longleftrightarrow \quad f(x)+f(\bar{x}) \leq g(x)+g(\bar{x}) \quad \text { and } \\
&(9.2) \max \{f(x) ; f(\bar{x})\} \leq \max \{g(x) ; g(\bar{x})\} \quad \forall x \in H, \\
&(9.3) \quad \Longleftrightarrow \quad \int_{\mathbb{R}^{n}} f_{H} h_{H} d x \leq \int_{\mathbb{R}^{n}} g_{H} h_{H} d x \quad \forall h \in L_{+}^{\infty}\left(\mathbb{R}^{n}\right), \\
&(9.4) \quad \Longleftrightarrow \quad j(f) \prec_{H} j(g) \quad \text { for all Lipschitz continuous Young functions } j .
\end{aligned}
$$

Proof. $(9.2) \Rightarrow(9.3)$ :

From (9.2) we deduce that

$f_{H}(x) h_{H}(x)+f_{H}(\bar{x}) h_{H}(\bar{x}) \leq g_{H}(x) h_{H}(x)+g_{H}(\bar{x}) h_{H}(\bar{x}) \quad \forall x \in H, h \in L_{+}^{\infty}\left(\mathbb{R}^{n}\right)$.

An integration over $H$ yields (9.3).

$$
(9.3) \Rightarrow(9.1) \text { : }
$$

By the Hardy-Littlewood inequality (3.10) we have $\int f h \leq \int f_{H} h_{H}$.

$$
(9.1) \Rightarrow(9.2) \text { : }
$$

Choosing in $(9.1) h=\delta(x, \cdot)+\delta(\bar{x}, \cdot)$ or $h=\delta(x, \cdot)$ for an arbitrary $x \in \mathbb{R}^{n}$ gives (9.2).

$(9.1) \Leftrightarrow(9.4)$ :

If $a_{i}, b_{i}, i=1,2$, are nonnegative numbers satisfying

$$
a_{1}+b_{1} \leq a_{2}+b_{2} \text { and } \max \left\{a_{1} ; b_{1}\right\} \leq \max \left\{a_{2} ; b_{2}\right\},
$$

and $j$ is a Young-function, then one can easily derive that

$$
j\left(a_{1}\right)+j\left(b_{1}\right) \leq j\left(a_{2}\right)+j\left(b_{2}\right) .
$$

Thus, because of the equivalence of (9.1) and (9.2), from (9.1) follows (9.4). Clearly (9.1) is a special case of (9.4).

Remark 9.1. Of course, if $f, g \in L_{+}^{p}\left(\mathbb{R}^{n}\right)(1<p \leq+\infty)$, then we may take $h$ in $L_{+}^{p^{\prime}}\left(\mathbb{R}^{n}\right)$ in (9.1), (9.3) while in (9.4) we may relax the assumption that $j^{\prime}$ is bounded by assuming $j(z) \leq C\left(1+z^{p}\right)$ if $p<+\infty$ (and nothing if $p=+\infty$ ).

As one can see from simple examples, the polarization of a domain may be disconnected, i.e. need not be a domain. For this reason we prefer to state our boundary and initial value problems in open sets instead of domains.

The next definition will be useful in order to simplify some notations in our proofs.

Definition 9.1. Let $\Omega \subset \mathbb{R}^{n}$ be a bounded open set, $c \in \mathbb{R}_{0}^{+}$and $f \in L_{+}^{2}(\Omega)$. We say that $u$ solves the problem $\mathbb{B}_{1}(\Omega, c, f)$ if $u$ is the solution of the following boundary value problem:

$$
u \in W_{0}^{1,2}(\Omega), \quad-\Delta u+c u=f \quad \text { in } \Omega .
$$


The following simple comparison theorem will play a central role in some proofs of the next section.

Theorem 9.1. Let $H$ be some halfspace, $\Omega \subset \mathbb{R}^{n}$ a bounded open set, $c \in \mathbb{R}_{0}^{+}$, $f \in L_{+}^{2}(\Omega), g \in L_{+}^{2}\left(\Omega_{H}\right)$ and assume that $f \prec_{H} g$ and $g=g_{H}$. Let $u$ and $v$ be the solutions of problems $\mathbb{B}_{1}(\Omega, c, f)$ and $\mathbb{B}_{1}\left(\Omega_{H}, c, g\right)$, respectively. Then

$$
u \prec_{H} v
$$

and

$$
v=v_{H}
$$

Furthermore we have

$$
v(x)-v(\bar{x}) \geq|u(x)-u(\bar{x})| \quad \forall x \in \Omega_{H} \cap H,
$$

and, if $u \in C_{\mathrm{loc}}^{1}(\Omega), v \in C_{\mathrm{loc}}^{1}\left(\Omega_{H}\right)$, then

$$
\frac{\partial v}{\partial n} \geq\left|\frac{\partial u}{\partial n}\right| \quad \text { on } \partial H \cap \Omega \quad(n: \text { interior normal }) .
$$

Proof. We set $G:=\{x \in H: \bar{x} \in \Omega\}$. Let us first assume that

$$
u \in C_{\mathrm{loc}}^{1}(\Omega) \cap C(\bar{\Omega}), \quad v \in C_{\mathrm{loc}}^{1}\left(\Omega_{H}\right) \cap C\left(\overline{\Omega_{H}}\right) .
$$

The proof of (9.6) consists of three steps.

1) Consider the function

$$
w_{1}(x):=u(x)+u(\bar{x})-v(x)-v(\bar{x}), \quad x \in \Omega_{H} \cap H .
$$

Let us assume that

$$
\sup _{H} w_{1}>0 .
$$

One verifies easily that $-\Delta w_{1}+c w_{1} \leq 0$ in $(G \backslash \bar{\Omega}) \cap H,(\Omega \backslash \bar{G}) \cap H$ and $(\Omega \cap G) \cap H$, respectively. Since $\left(\partial w_{1}\right) /(\partial \nu)=0$ on $\partial H \cap \Omega(\nu$ : normal to the hyperplane $\partial H)$, the maximum principle ensures that one of the following cases (i) or (ii) is satisfied:

$$
\begin{aligned}
& \sup _{H} w_{1}=\sup _{\partial G \cap \Omega} w_{1}, \\
& \sup _{H} w_{1}=\sup _{\partial \Omega \cap G} w_{1} .
\end{aligned}
$$

(i) We consider the function

$$
w_{2}(x):=u(x)-v(x), \quad x \in \Omega \cap H .
$$

Since $-\Delta w_{2}+c w_{2} \leq 0$ in $\Omega \cap H$ and $w_{2} \leq 0$ on $\partial \Omega \cap H, w_{2}$ attains its maximum value at some point $z_{1} \in \partial H \cap \Omega$. Thus

$$
\begin{aligned}
& w_{1}\left(z_{1}\right) \\
\left(\text { since } z_{1} \in \partial H \cap \Omega !\right) & =2 w_{2}\left(z_{1}\right) \\
\left(\text { maximality of } z_{1} !\right) & \geq 2 \sup _{\partial G \cap \Omega} w_{2} \\
\left(\text { since } w_{1}=w_{2} \text { on } \partial G \cap \Omega !\right) & =2 \sup _{\partial G \cap \Omega} w_{1},
\end{aligned}
$$

a contradiction.

(ii) We consider the function

$$
w_{3}(x):=u(\bar{x})-v(x), \quad x \in G .
$$


Since $-\Delta w_{3}+c w_{3} \leq 0$ in $G$ and $w_{3} \leq 0$ on $\partial G \cap H, w_{3}$ attains its maximum value at some point $z_{2} \in \partial H \cap \Omega$. Therefore

$$
\begin{array}{rlrl} 
& & w_{1}\left(z_{2}\right) \\
\left(\text { since } z_{2} \in \partial H \cap \Omega !\right) & = & 2 w_{3}\left(z_{2}\right) \\
\left(\text { maximality of } z_{2} !\right) & \geq 2 \sup _{\partial \Omega \cap G} w_{3} \\
\text { (since } \left.w_{1}=w_{3} \text { on } \partial \Omega \cap G !\right) & =2 \sup _{\partial \Omega \cap G} w_{1},
\end{array}
$$

a contradiction. Thus we have proved that

$$
w_{1}(x) \leq 0 \quad \forall x \in \Omega_{H} \cap H .
$$

2) We consider the function $w_{2}$ in $\Omega_{H} \cap H$.

We have $w_{2}(x)=2 w_{1}(x) \leq 0$ on $\partial H \cap \Omega$ and $w_{2}(x)=w_{1}(x) \leq 0$ on $\partial \Omega_{H} \cap H$. As $g \geq 0$, we have $w_{2}(x)=-v(x) \leq 0$ in $(G \backslash \bar{\Omega}) \cap H$. Since $-\Delta w_{2}+c w_{2} \leq 0$ in $(G \backslash \bar{\Omega}) \cap H$, the maximum principle tells us that

$$
w_{2} \leq 0 \quad \text { in } \Omega_{H} \cap H .
$$

3) We consider the function $w_{3}$ in $\Omega_{H} \cap H$.

We have $w_{3}(x)=2 w_{1}(x) \leq 0$ on $\partial H \cap \Omega, w_{3}(x)=w_{1}(x) \leq 0$ on $\partial \Omega_{H} \cap H$ and $w_{3}(x)=-v(x) \leq 0$ in $(\Omega \backslash \bar{G}) \cap H$. Since $-\Delta w_{3}+c w_{3} \leq 0$ in $G$, we conclude by the maximum principle that

$$
w_{3} \leq 0 \quad \text { in } \Omega_{H} \cap H .
$$

Now (9.6) follows from (9.11)-(9.13).

If we replace the second condition in (9.10) by the weaker assumption

$$
v \in C_{\mathrm{loc}}^{1}\left(\Omega_{H}\right),
$$

then

$$
\liminf _{z \rightarrow x} v(z) \geq 0, \quad x \in \partial \Omega_{H}
$$

and the arguments of the above steps $\mathbf{1})-\mathbf{3}$ ) hold with slight modifications.

In the general case we choose open sets $\Omega_{m}$ with smooth boundary, such that

$$
\Omega_{m} \Subset \Omega_{m+1}, \quad m=1,2, \ldots, \quad \text { and } \bigcup_{m} \Omega_{m}=\Omega,
$$

and functions $f_{m}:=f-(f-m)_{+}, g_{m}:=g-(g-2 m)_{+}, m=1,2, \ldots$ Then let $u_{m}$ and $v_{m}$ be the solutions of problems $\mathbb{B}_{1}\left(\Omega_{m}, c, f_{m}\right)$ and $\mathbb{B}_{1}\left(\left(\Omega_{m}\right)_{H}, c, g_{m}\right)$, respectively. Since $f_{m} \in L^{\infty}(\Omega)$ and $g_{m} \in L^{\infty}\left(\Omega_{H}\right)$, we have concluded $u_{m} \in$ $C_{\mathrm{loc}}^{1}\left(\Omega_{m}\right) \cap C\left(\overline{\Omega_{m}}\right)$ and $v_{m} \in C_{\mathrm{loc}}^{1}\left(\left(\Omega_{m}\right)_{H}\right)$ by the imbedding theorems. Since $f_{m} \prec_{H} g_{m}$, it follows then that $u_{m} \prec_{H} v_{m}, m=1,2, \ldots$ Furthermore, we have $f_{m} \rightarrow f$ in $L^{2}(\Omega), g_{m} \rightarrow g$ in $L^{2}\left(\Omega_{H}\right)$ and $\bigcup_{m}\left(\Omega_{m}\right)_{H}=\Omega_{H}$. By applying Lemma A (Appendix) this yields $u_{m} \rightarrow u$ in $W_{0}^{1,2}(\Omega)$ and $v_{m} \rightarrow v$ in $W_{0}^{1,2}\left(\Omega_{H}\right)$, and (9.6) follows.

The assertion (9.7) follows by applying (9.6) with $g=f_{H}$ and $u=\sigma_{H}(v)$.

Inequalities (9.8) are proved in a similar way. Clearly (9.9) is a limit case of (9.8).

Analogous to the above proof one can derive similar pointwise inequalities for Green's functions (see [So2]). On the other hand these inequalities can be seen as special cases of (9.6),(9.7) via a limit process. 
Corollary 9.1. Let $G$ and $\tilde{G}$ denote the Green's functions for the Dirichlet problems $\mathbb{B}_{1}(\Omega, c, \cdot)$ and $\mathbb{B}_{1}\left(\Omega_{H}, c, \cdot\right)$, respectively. Then

$$
G(\cdot, z) \prec_{H} \tilde{G}(\cdot, z), \quad G(\cdot, \bar{z}) \prec_{H} \tilde{G}(\cdot, z) \quad \forall z \in H,
$$

and

$$
\tilde{G}(x, z)-\tilde{G}(\bar{x}, z) \geq \max \{|G(x, z)-G(\bar{x}, z)| ;|G(x, \bar{z})-G(\bar{x}, \bar{z})|\} \quad \forall x, z \in H .
$$

Proof. We denote by $\delta(x, z)$ the usual Delta distribution $\left(x, z \in \mathbb{R}^{n}\right)$. Then formally $G(\cdot, z)$ is the solution of the problem $\mathbb{B}_{1}(\Omega, c, \delta(\cdot, z)) \quad \forall z \in \Omega$ and $\tilde{G}(\cdot, z)$ is the solution of the problem $\mathbb{B}_{1}\left(\Omega_{H}, c, \delta(\cdot, z)\right) \forall z \in \Omega_{H}$. Since

$$
(\delta(\cdot, z))_{H}=(\delta(\cdot, \bar{z}))_{H}=\delta(\cdot, z) \quad \forall z \in H,
$$

the Green's functions $G(\cdot, z)$, (respectively $G(\cdot, \bar{z}))$, and $\tilde{G}(\cdot, z)$ can be approximated by "polarized pairs" of regular Dirichlet problems exactly as in Theorem 9.1. We leave the details to the reader.

Next we will investigate comparison results for so-called nonnegative minimal solutions of boundary value problems.

Definition 9.2. Let $\Omega \subset \mathbb{R}^{n}$ be a bounded open set, $c \in \mathbb{R}_{0}^{+}, f \in L_{+}^{2}(\Omega)$ and $\gamma: \mathbb{R}_{0}^{+} \rightarrow \mathbb{R}_{0}^{+}$be a continuous and nondecreasing function. We will say that $\underline{u}$ is a solution of problem $\mathbb{B}_{2}(\Omega, c, \gamma, f)$, if $\underline{u}$ is the nonnegative minimal solution of the following boundary value problem:

$$
u \in W_{0}^{1,2}(\Omega), \quad u \geq 0, \quad-\Delta u+c u=\gamma(u)+f \quad \text { in } \Omega,
$$

that is,

(i) $\underline{u}$ is a solution of the problem (9.16), and

(ii) $0 \leq \underline{u} \leq u$ for all other solutions $u$ of (9.16).

Remark 9.2. Nonnegative minimal solutions describe stable ("ground") equilibrium states in heat conduction. We mention some properties of the solutions of the problems in Definition 9.1 (for further information see [Ke]).

1) The nonnegative minimal solutions of the boundary value problems (9.16) (whenever they exist!) are unique, and they can be generated by monotone iteration.

If $\underline{u}$ solves the problem $\mathbb{B}_{2}(\Omega, c, \gamma, f), u_{0} \equiv 0$, and $u_{m}$ are solutions of the problems $\mathbb{B}_{1}\left(\Omega, c, \gamma\left(u_{m-1}\right)+f\right), m=1,2, \ldots$, then the sequence $\left\{u_{m}\right\}$ is monotone increasing and

$$
\lim _{m \rightarrow \infty} u_{m}(x)=\underline{u}(x) \quad \text { for a.e. } x \in \Omega .
$$

2) The notion of nonnegative minimal solution overlaps with some cases of uniquely solvable boundary value problems.

Let $\Omega, c$ and $f$ be as in Definition 9.2, let $\gamma: \mathbb{R}_{0}^{+} \rightarrow \mathbb{R}_{0}^{+}$, and assume that $\gamma$ has a difference quotient which is bounded below, i.e. we have, for some number $k \geq 0$,

$$
\frac{\gamma(s)-\gamma(t)}{s-t} \geq-k \quad \forall s, t \geq 0 .
$$

Further assume that the boundary value problem (9.16) admits a unique solution $u$. Then it follows from $\mathbf{1}$ ) that $u$ coincides with the solution of problem $\mathbb{B}_{2}(\Omega, c+k, \tilde{\gamma}, f)$ where $\tilde{\gamma}(t):=\gamma(t)+k t, t \geq 0$. 
Note that boundary value problem (9.16) is uniquely solvable, if $\gamma$ is a decreasing function for instance.

Theorem 9.2. Let $\Omega, c, f, g$ and $H$ be as in Theorem 9.1, and let $\gamma$ be a Young function. Assume that $u$ and $v$ are solutions of the problems $\mathbb{B}_{2}(\Omega, c, \gamma, f)$ and $\mathbb{B}_{2}\left(\Omega_{H}, c, \gamma, g\right)$, respectively. Then conclusions (9.6) and (9.7) of Theorem 9.1 hold.

Proof. According to Remark 9.2 we approximate $u$ and $v$ by the solutions $u_{m}$ and $v_{m}$ of problems $\mathbb{B}_{1}\left(\Omega, c, \gamma\left(u_{m-1}\right)+f\right)$ and $\mathbb{B}_{1}\left(\Omega_{H}, c, \gamma\left(v_{m-1}\right)+g\right)$, respectively,

$m=1,2, \ldots, u_{0} \equiv v_{0} \equiv 0$. Assume that $u_{m} \prec_{H} v_{m}$ for some $m \in \mathbb{N}$. (For $m=0$ this is true.) Then by Lemma 9.1, (9.4), it follows that $\left(\gamma\left(u_{m}\right)+f\right) \prec_{H}$ $\left(\gamma\left(v_{m}\right)+g\right)$. In view of Theorem 9.1 this yields $u_{m+1} \prec_{H} v_{m+1}$, and we conclude by induction.

It is natural to ask in which situations we have $u_{H}=v$ in Theorem 9.2. An answer is given by the following Theorem 9.3. The reader verifies easily that we cannot drop the condition that $\Omega$ is connected.

Theorem 9.3. Let $\Omega, c, f, g, H, \gamma, u, v$ be as in Theorem 9.2, and assume that $\Omega$ is a domain with $\Omega \neq \sigma_{H}(\Omega)$ and $\|f\|_{2}>0$. Let one of the following situations be valid:

(i) $\Omega \neq \Omega_{H}$;

(ii) $\Omega=\Omega_{H}$ and $f \neq g$;

(iii) $\sigma_{H}(\Omega)=\Omega_{H}$ and $\sigma_{H}(f) \neq g$.

Then

$$
\begin{aligned}
u(x)+u(\bar{x}) & <v(x)+v(\bar{x}) \quad \text { and } \\
\max \{u(x) ; u(\bar{x})\} & <\max \{v(x) ; v(\bar{x})\} \quad \text { for a.e. } x \in \Omega .
\end{aligned}
$$

If the solutions $u, v$ are continuous in $\Omega$, respectively $\Omega_{H}$, then inequalities (9.18) are satisfied for all points $x \in \Omega$.

Furthermore, if $j$ is any Young function satisfying

$$
\int_{\Omega_{H}} j(v) d x>0,
$$

then

$$
\int_{\Omega} j(u) d x<\int_{\Omega_{H}} j(v) d x .
$$

Proof. We use the notations of the proof of Theorem 9.1. First recall that by Theorem 9.2 we have $u \prec_{H} v$ and thus - by Lemma 9.1-also $(\gamma(u)+f) \prec_{H}$ $(\gamma(v)+g)$.

Now we investigate the cases (i)-(iii).

(i) Since $u \geq 0$, it follows that $-\Delta w_{2}+c w_{2} \leq 0$ and $-\Delta w_{3}+c w_{3} \leq 0$ weakly in $\Omega_{H} \cap H$. Since also $w_{2} \leq 0, w_{3} \leq 0$ in $\Omega_{H} \cap H, w_{2}<0$ in $(G \backslash \bar{\Omega}) \cap H$ and $w_{3}<0$ in $(\Omega \backslash \bar{G}) \cap H$, the strong maximum principle yields

$$
w_{2}<0 \text { and } w_{3}<0 \text { in } \Omega_{H} \cap H,
$$

which gives the second inequality of (9.18). 
From (9.21) we see that $w_{1}=w_{2}<0$ in $(\Omega \backslash G) \cap H, w_{1}=w_{3}<0$ in $(G \backslash \Omega) \cap H$. Since $w_{1} \leq 0$ and $-\Delta w_{1}+c w_{1} \leq 0$ in $\Omega \cap G \cap H$, the strong maximum principle gives

$$
w_{1}<0 \quad \text { in } \Omega_{H} \cap H,
$$

which is the first inequality of (9.18).

(ii) Analogously as above we can conclude that $w_{3}<0$ in $\Omega \cap H$. Further we have $w_{2} \leq 0$ and $-\Delta w_{2}+c w_{2} \leq f-g \leq 0$ in $\Omega \cap H$. Since

$$
\int_{\Omega \cap H}(f-g) d x<0,
$$

the strong maximum principle yields $w_{2}<0$ in $\Omega \cap H$. Then we can argue as in case (i) to obtain that $w_{1}<0$, and (9.18) follows.

(iii) In this case the assertion follows by replacing $u$ in (ii) by the reflected function $\sigma_{H}(u)$.

Finally let $j$ be a Young function satisfying (9.19). Then it follows that $j(v)>0$ on a subset $N$ of $\Omega_{H} \cap H$ of positive measure. From (9.18) we infer

$$
j\left(u_{H}(x)\right)+j\left(u_{H}(\bar{x})\right) \leq j(v(x))+j(v(\bar{x})) \quad \text { for a.e. } x \in H \cap \Omega_{H},
$$

whereby these inequalities are strict on $N$. This leads after an integration over $H$ to the second assertion (9.20).

Since the proofs of the comparison theorems, Theorems 9.1, 9.2, depend only on the maximum principle, we can derive similar results for parabolic problems. The proof presented here is based on an approximation scheme involving solutions of some elliptic problems. This idea was used in ALT3 to show comparison results via Schwarz symmetrization. As we will see in the next section, this method works also for other types of rearrangements.

Let us introduce some notations.

Definition 9.3. Let $\Omega, c$ be as in Definition 9.2, let $T>0, f \in L_{+}^{2}(\Omega \times(0, T))$, $\varphi \in L_{+}^{2}(\Omega)$, and let $\gamma: \mathbb{R}_{0}^{+} \rightarrow \mathbb{R}_{0}^{+}$be a globally Lipschitzian function. We say that $u$ solves the problem $\mathbb{I}(\Omega, T, c, \gamma, f, \varphi)$ if $u$ is a solution of the following initial boundary value problem:

$$
\begin{aligned}
& u \in L^{2}\left(0, T ; W_{0}^{1,2}(\Omega)\right) \cap C\left([0, T] ; L^{2}(\Omega)\right), \frac{\partial u}{\partial t} \in L_{2}\left([0, T] ; L^{2}(\Omega)\right), \\
& u_{t}-\Delta u+c u=\gamma(u)+f \quad \text { in } \Omega \times(0, T), \\
& u(x, 0)=\varphi(x) \quad \text { in } \Omega .
\end{aligned}
$$

Remark 9.3. Under the above conditions problem $\mathbb{I}(\Omega, T, c, \gamma, f, \varphi)$ has a unique nonnegative solution which can be approximated by the so-called method of discretization in time (see $[\mathrm{Kac}]$ ).

We choose some number $N \in \mathbb{N}$ and divide the interval $(0, T)$ into $N$ subintervals $\left[t_{i-1}, t_{i}\right]$, where $t_{i}=(i T) / N$, and we set

$$
f_{i}(x):=\frac{T}{N} \int_{t_{i-1}}^{t_{i}} f(x, s) d s, \quad i=1, \ldots, N .
$$

For $i=1, \ldots, N$, let $u_{i}$ be the solution of the problem

$$
\mathbb{B}_{1}\left(\Omega, c+(N / T), \gamma\left(u_{i-1}\right)+f_{i}+(N / T) u_{i-1}\right),
$$


provided $u_{i-1}$ is known and $u_{0} \equiv \varphi$. Then by setting

$$
\begin{aligned}
& u^{N}(x, t):=u_{i-1}(x)+\left(t-t_{i-1}\right) \frac{N}{T}\left(u_{i}(x)-u_{i-1}(x)\right) \\
& \quad \text { for } t_{i-1} \leq t \leq t_{i}, i=1 \ldots, N,
\end{aligned}
$$

we have (see [Kac, Theorem 2.2.4, p.42 ff.])

$$
\begin{aligned}
& u^{N}(\cdot, t) \rightarrow u(\cdot, t) \quad \text { weakly in } W_{0}^{1,2}(\Omega) \quad \forall t \in(0, T), \\
& \frac{\partial u^{N}}{\partial t} \rightarrow \frac{\partial u}{\partial t} \quad \text { weakly in } L^{2}\left([0, T] ; L^{2}(\Omega)\right),
\end{aligned}
$$

and

$$
u^{N} \longrightarrow u \quad \text { in } C\left(0, T ; L^{2}(\Omega)\right) .
$$

Theorem 9.4. Let $\Omega, T, c, \gamma$ be as in Definition 9.3, and let $\gamma$ be convex, let $H$ be some halfspace, let $f \in L_{+}^{2}(\Omega \times(0, T)), g \in L_{+}^{2}\left(\Omega_{H} \times(0, T)\right), \varphi \in L_{+}^{2}(\Omega)$ and $\psi \in L_{+}^{2}\left(\Omega_{H}\right)$ with $\varphi \prec_{H} \psi$ and $f(\cdot, t) \prec_{H} g(\cdot, t) \quad \forall t \in(0, T)$. Let $u$ and $v$ be solutions of the problems $\mathbb{I}(\Omega, T, c, \gamma, f, \varphi)$ and $\mathbb{I}\left(\Omega_{H}, T, c, \gamma, g, \psi\right)$, respectively. Then

$$
u(\cdot, t) \prec_{H} v(\cdot, t) \quad \forall t \in(0, T)
$$

and

$$
v(\cdot, t)=v_{H}(\cdot, t) \quad \forall t \in(0, T) .
$$

Proof. Let $u_{i}$ be as in Remark 9.3, and let $v_{i}$ be defined analogously by replacing $\Omega, f$ and $\varphi$ by $\Omega_{H}, g$ and $\psi$, respectively, $i=0,1, \ldots, N$. By the assumptions we have $u_{0} \prec_{H} v_{0}$. If $N$ is large enough, then $\gamma(s)+N s / T$ is increasing and convex in $s$, and we have $f_{i} \prec_{H} g_{i}, i=1, \ldots, N$, by (9.24). Therefore we can argue as in the proof of Theorem 9.2, and obtain that $u_{i} \prec_{H} v_{i}, i=1, \ldots, N$. By (9.25) it follows that $u^{N} \prec_{H} v^{N}$. Then passing to the limit as $N \rightarrow+\infty$ we conclude.

\section{COMPARISOn ReSults FOR SYMmetrizAtions}

In the following we let $*$ denote any $(k, n)$-Steiner symmetrization $(1 \leq k \leq n)$, and use the notations of section 6 .

As in the previous section we introduce a partial order " $\prec$ ".

For functions $f, g \in L_{+}^{1}\left(\mathbb{R}^{n}\right)$ we write

$$
f \prec^{*} g \Longleftrightarrow \int_{\mathbb{R}^{n}} f h d x \leq \int_{\mathbb{R}^{n}} g^{*} h^{*} d x \quad \forall h \in L_{+}^{\infty}\left(\mathbb{R}^{n}\right) .
$$

Remark 10.1. The partial order " ${ }^{*}$ " was introduced in ALT1. The following equivalences hold (for proofs see [ALT2]).

$$
\begin{aligned}
& f \prec^{*} g \\
\Longleftrightarrow & \int_{\mathbb{R}^{n}} f^{*} h^{*} d x \leq \int_{\mathbb{R}^{n}} g^{*} h^{*} d x \quad \forall h \in L_{+}^{\infty}\left(\mathbb{R}^{n}\right), \\
\Longleftrightarrow & j(f) \prec^{*} j(g) \quad \forall \text { Lipschitz-continuous Young functions } j, \\
\Longleftrightarrow & \int_{\{|z| \leq r\}} f^{*}\left(x^{\prime}, z\right) d z \leq \int_{\{|z| \leq r\}} g^{*}\left(x^{\prime}, z\right) d z \quad \forall r>0, x^{\prime} \in \mathbb{R}^{n-k} .
\end{aligned}
$$

Corollary 10.1. Let $H \in \mathcal{H}, f, g \in L_{+}^{1}\left(\mathbb{R}^{n}\right)$ and $f \prec_{H} g$. Then we have $f \prec^{*} g$. 
Proof. By (6.6) and (3.10) we have

$$
\int_{\mathbb{R}^{n}} f h d x \leq \int_{\mathbb{R}^{n}} g_{H} h_{H} d x \leq \int_{\mathbb{R}^{n}} g^{*} h^{*} d x \quad \forall h \in L_{+}^{\infty}\left(\mathbb{R}^{n}\right) .
$$

The following result was proved in various ways (see e.g. [Ta1], [Ban], [ADLT, ALT2] and [Bae2]).

The present proof seems to be the most elementary one. It is based on an approximation of symmetrizations by sequences of polarizations and on comparison theorem 9.1.

Theorem 10.1. Let be $\Omega, c, f$ and $\gamma$ be as in Theorem 9.2, let $g \in L_{+}^{2}\left(\Omega^{*}\right)$ with $f \prec^{*} g$ and $g=g^{*}$, and let $u$ and $v$ be the solutions of the problems $\mathbb{B}_{2}(\Omega, c, \gamma, f)$ and $\mathbb{B}_{2}\left(\Omega^{*}, c, \gamma, g\right)$, respectively. Then

$$
u \prec * v
$$

and

$$
v=v^{*} .
$$

Proof. The proof consists of two steps.

1) First assume that $\gamma \equiv 0$.

Let $\tilde{v}$ denote the solution of the problem $\mathbb{B}_{1}\left(\Omega^{*}, c, f^{*}\right)$. The maximum principle tells us that $v=v^{*}$ and $\tilde{v}=\tilde{v}^{*}$. Furthermore, let $h$ be an arbitrary function in $L_{+}^{2}\left(\Omega^{*}\right)$ satisfying $h=h^{*}$, and let $w$ be the solution of problem $\mathbb{B}_{1}\left(\Omega^{*}, c, h\right)$. Since again $w=w^{*}$ and $w \geq 0$, we find after partial integration that

$$
\int_{\Omega^{*}} \tilde{v} h^{*} d x=\int_{\Omega^{*}} w f^{*} d x \leq \int_{\Omega^{*}} w g d x=\int_{\Omega^{*}} v h^{*} d x
$$

which means that

$$
\tilde{v} \prec^{*} v .
$$

Next let $\Omega^{\prime}$ be a domain with $\Omega^{\prime} \Subset \Omega$. By Lemma 7.1 we find polarizations $P_{m}$, with corresponding halfspaces $H_{i} \in \mathcal{H}_{0}, i=1, \ldots, m_{0}$, such that $\bigcirc_{i=1}^{m_{0}} P_{i}\left(\Omega^{\prime}\right) \Subset \Omega^{*}$. Clearly we have $\left(\bigcirc_{i=1}^{m_{0}} P_{i} f\right)^{*}=f^{*}$. Then we can find further polarizations $P_{i}$, with corresponding halfspaces $H_{i} \in \mathcal{H}_{0}, i=m_{0}+1, m_{0}+2, \ldots$, such that

$$
f_{m}:=\bigcirc_{i=1}^{m} P_{i} f \longrightarrow f^{*} \quad \text { in } L^{2}(\Omega) .
$$

Note that by monotonicity we have

$$
\Omega_{m}^{\prime}:=\bigcirc_{i=1}^{m} P_{i}\left(\Omega^{\prime}\right) \Subset \Omega^{*} \quad \forall m \geq m_{0} .
$$

Let $u^{\prime}$ and $u_{m}^{\prime}$ be the solutions of the problems $\mathbb{B}_{1}\left(\Omega^{\prime}, c, f\right)$ and $\mathbb{B}_{1}\left(\Omega_{m}^{\prime}, c, f_{m}\right)$, respectively, $m=1,2, \ldots$. Applying Theorem 9.1 we conclude that

$$
u^{\prime} \prec_{H_{1}} u_{1}^{\prime} \prec_{H_{2}} u_{2}^{\prime} \prec_{H_{3}} \ldots
$$

Since the functions $u_{m}^{\prime}$ are equibounded in $W^{1,2}\left(\mathbb{R}^{n}\right)$ and $u_{m}^{\prime} \in W_{0}^{1,2}\left(\Omega^{*}\right)$ for $m \geq$ $m_{0}$, we find a function $w \in W_{0}^{1,2}\left(\Omega^{*}\right)$ and a subsequence $u_{m^{\prime}}^{\prime}$ such that

$$
u_{m^{\prime}}^{\prime} \rightarrow w \quad \text { weakly in } W_{0}^{1,2}\left(\Omega^{*}\right) \text {. }
$$


By passing again to a subsequence, if necessary, we have that

$$
u_{m^{\prime}}^{\prime} \longrightarrow w \quad \text { in } L^{2}\left(\mathbb{R}^{n}\right)
$$

and

$$
\lim _{m^{\prime} \rightarrow \infty} u_{m^{\prime}}^{\prime}(x)=w(x) \quad \text { for a.e. } x \in \Omega^{*} .
$$

Together with (10.8) and Corollary 10.1 this leads to

$$
u^{\prime} \prec^{*} w .
$$

Now let $\varphi \in C_{0+}^{\infty}\left(\Omega^{*}\right)$. Since the functions $u_{m}^{\prime}$ are nonnegative, we obtain that

$$
\begin{aligned}
\int_{\Omega^{*}} \nabla w \nabla \varphi d x & \longleftarrow \int_{\Omega_{m^{\prime}}^{\prime}} \nabla u_{m^{\prime}}^{\prime} \nabla \varphi d x \\
& \leq \int_{\Omega_{m^{\prime}}^{\prime}}\left(-c u_{m^{\prime}}^{\prime}+f_{m^{\prime}}\right) \varphi d x \longrightarrow \int_{\Omega^{*}}\left(-c w+f^{*}\right) \varphi d x
\end{aligned}
$$

as $m^{\prime} \rightarrow \infty$, i.e $w$ is a weak subsolution to the problem $\mathbb{B}_{1}\left(\Omega^{*}, c, f^{*}\right)$. Together with (10.7) and (10.9) this means that $u^{\prime} \prec^{*} v$.

Now we choose open bounded sets $\Omega^{k}$ such that $\Omega^{k} \Subset \Omega^{k+1}, k=1,2, \ldots$, and $\bigcup_{k} \Omega^{k}=\Omega$. Let $u^{k}$ denote the solution of the problem $\mathbb{B}_{1}\left(\Omega^{k}, c, f\right), k=1,2, \ldots$. By the above considerations we have $u^{k} \prec^{*} v, k=1,2, \ldots$, and by Lemma A (Appendix) the sequence $u^{k}$ converges to $u$ in $L^{2}(\Omega)$. This proves the assertions in the case under consideration.

2) Next let $\gamma \neq 0$.

According to Remark 9.2 we approximate $u$ and $v$ by the solutions $u_{m}$ and $v_{m}$ of the problems $\mathbb{B}_{1}\left(\Omega, c, \gamma\left(u_{m-1}\right)+f\right)$ and $\mathbb{B}_{1}\left(\Omega^{*}, c, \gamma\left(v_{m-1}\right)+g\right)$, respectively $\left(u_{0} \equiv\right.$ $\left.v_{0} \equiv 0, m=1,2, \ldots\right)$. Assume that we had proved that $u_{m} \prec^{*} v_{m}$ for some $m$. (For $m=0$ this is trivial.) Then by Remark 10.1 we obtain that $\gamma\left(u_{m}\right)+f \prec^{*} \gamma\left(v_{m}\right)+g$. By part 1) of the proof this means that also $u_{m+1} \prec^{*} v_{m+1}$, and we conclude by induction.

Remark 10.2. 1) From (10.5) we obtain via Remark 10.1 the estimates

$$
\int_{\Omega} j(u) d x \leq \int_{\Omega^{*}} j(v) d x \quad \forall \text { Young functions } j,
$$

and in particular

$$
\|u\|_{p} \leq\|v\|_{p} \quad \forall p \in[1,+\infty]
$$

if the above integrals converge.

2) As was pointed out by Baernstein [Bae2], in the case that $k=n$ (Schwarzsymmetrization) and $c=0, \gamma \equiv 0$, one can exploit the radial symmetry of the solution $v$ to derive from (10.5) the sharper inequality

$$
u^{*} \leq v \quad \text { in } \Omega^{*} \text {. }
$$

This result was also proved by other authors in various ways (see Ta1, Ban]).

The following theorem is due to Bandle (see [Ban, Theorem 10.4]).

Theorem 10.2. Let $u, v$ be as in Theorem 10.1, where * denotes Schwarz symmetrization, and assume that $c=0$ and $\gamma: \mathbb{R}_{0}^{+} \rightarrow \mathbb{R}_{0}^{+}$is a continuous and nondecreasing function, but not nessecarily convex. Then (10.12) holds. 
Proof. We can argue analogously to part 2) of the proof of Theorem 10.1 by replacing the relations " $\prec$ " by " $\leq$ ", and by making use of the conclusion (10.12) of Remark 10.2,2) instead of (10.5).

One might ask under which conditions the equality holds in inequalities (10.10), and believe that equality is possible only - roughly speaking - in a symmetric situation. Indeed this belief is proved true in the cases of spherical symmetrization (see [ESh]) and Schwarz symmetrization (see [Kes1] Kes3]). We prove a similar result for the $(k, n)$-Steiner symmetrizations. As in the uniqueness Theorem 9.3, we restrict ourselves to the case where $\Omega$ is a domain.

Theorem 10.3. Let $\Omega, c, f, g, \gamma, u, v$ be as in Theorem 10.1 and assume that $\Omega$ is a domain. Assume that there is some Lipschitz continuous Young function $j$ which satisfies

$$
\int_{\Omega} j(u) d x=\int_{\Omega^{*}} j(v) d x>0 .
$$

Then we have $\Omega=\Omega^{*}$ and $f=g$ modulo some translation in a direction orthogonal to $\Sigma$.

Proof. Assume that $\Omega^{*}$ is not a translation of $\Omega$ in a direction orthogonal to $\Sigma$. Then by Lemma $6.3,(6.9)$ we can find a halfspace $H \in \mathcal{H}$ such that $\Omega \neq \Omega_{H}$ and $\sigma_{H}(\Omega) \neq \Omega_{H}$. Then, if $w$ is the solution of problem $\mathbb{B}_{2}\left(\Omega_{H}, c, \gamma, f_{H}\right)$, we conclude by Theorem 9.3 that

$$
\int_{\Omega} j(u) d x<\int_{\Omega_{H}} j(w) d x .
$$

Further, since $\left(\Omega_{H}\right)^{*}=\Omega^{*}$ and $\left(f_{H}\right)^{*}=f^{*}$, we have also $w \prec^{*} v$. By Remark 10.2 this means that

$$
\int_{\Omega_{H}} j(w) d x \leq \int_{\Omega^{*}} j(v) d x
$$

which together with (10.14) contradicts (10.13). Thus $\Omega=\Omega^{*}$ modulo a translation in some direction orthogonal to $\Sigma$. Without loss of generality we may assume that $\Omega=\Omega^{*}$.

Now assume that $f \neq f^{*}$. By Lemma 6.4 there is a halfspace $H \in \mathcal{H}_{0}$ such that $f \neq f_{H}$, and we can argue as before to derive a contradiction to (10.13).

Thus we have $f=f^{*}$ and it remains to show that $f^{*}=g$.

Assume that this is not true. We set $\tilde{f}:=\gamma(u)+f$ and $\tilde{g}:=\gamma(v)+g$. Since $u=u^{*}, v=v^{*}$ and $u \prec^{*} v$, it follows by Remark 10.1 that

$$
\tilde{f} \prec^{*} \tilde{g}, \tilde{f}=\tilde{f}^{*} \neq \tilde{g}^{*}=\tilde{g},
$$

and also

$$
\int_{\{|z|<|y|\}}\left(\tilde{f}\left(x^{\prime}, z\right)-\tilde{g}\left(x^{\prime}, z\right)\right) d z \leq 0 \quad \forall\left(x^{\prime}, y\right) \in \Omega,
$$

where this last inequality must be strict on a subset of $\Omega$ of positive measure. Now let $h$ be an arbitrary function in $L_{+}^{2}(\Omega)$ satisfying $h=h^{*} \neq 0$. Then, if $w$ is the 
solution of problem $\mathbb{B}_{1}(\Omega, c, h)$, we conclude that $w=w^{*}$. Moreover, the strong maximum principle even yields

$$
\left|\nabla_{y} w(x)\right|>0 \quad \text { a. e. in } \Omega .
$$

(Here $\nabla_{y}$ denotes the gradient with respect to $y$.) Now after some partial integrations and by using (10.15) and (10.16) we obtain

$$
\begin{aligned}
& \int_{\Omega}(u-v) h d x=\int_{\Omega} w(\tilde{f}-\tilde{g}) d x \\
& =\quad\left(k c_{k}\right)^{-1} \int_{\Omega}|y|^{1-k}\left|\nabla_{y} w\left(x^{\prime}, y\right)\right|\left(\int_{\{|z|<|y|\}}\left(\tilde{f}\left(x^{\prime}, z\right)-\tilde{g}\left(x^{\prime}, z\right)\right) d z\right) d x^{\prime} d y<0, \\
& \quad\left(c_{k}: \quad \text { volume of the } k \text {-dimensional unit ball }\right) .
\end{aligned}
$$

Since $j^{\prime}$ is nondecreasing and $u=u^{*}$, we have $j^{\prime}(u)=\left(j^{\prime}(u)\right)^{*}$ (see (3.6)), and in view of (10.13) it follows that $j^{\prime}(u) \neq 0$. Therefore we may take $h=j^{\prime}(u)$ in (10.17). Because of the convexity of $j$ we get then

$$
\int_{\Omega}(j(u)-j(v)) d x \leq \int_{\Omega} j^{\prime}(u)(u-v) d x<0,
$$

a contradiction. The theorem is proved.

The following analogue of Theorem 9.4 for symmetrizations was proved in ALT2. Our proof repeats the proof of Theorem 9.4 with obvious changes.

Theorem 10.4. Let $\Omega, c, T, f, \gamma, \varphi$ and $u$ be as in Theorem 9.4, and let $g \in$ $L_{+}^{2}\left(\Omega^{*} \times(0, T)\right), \psi \in L_{+}^{2}\left(\Omega^{*}\right)$ with $f(\cdot, t) \prec^{*} g(\cdot, t)$ and $g(\cdot, t)=g(\cdot, t)^{*} \forall t \in(0, T)$, and $\varphi \prec^{*} \psi, \psi=\psi^{*}$. Let $v$ be the solution of problem $\mathbb{I}\left(\Omega^{*}, T, c, \gamma, g, \psi\right)$. Then

$$
u(\cdot, t) \prec^{*} v(\cdot, t) \quad \forall t \in(0, T)
$$

and

$$
v(\cdot, t)=v^{*}(\cdot, t) \quad \forall t \in(0, T) .
$$

Proof. We can proceed exactly as in the proof of Theorem 9.4 by replacing the polarization by the symmetrization and by making use of Remark 10.1, (10.3), and Theorem 10.1 instead of Lemma 9.1, (9.3) and Theorem 9.1, respectively.

Remark 10.3. The results of sections 9 and 10 remain true if one replaces the Laplacian by more general elliptic operators (see ADLT], [ALT2] and [Bae2]).

1) First consider the situation of Theorems 9.1-9.4. Let the halfspace $H$ take the form $\{y>0\}$, where $\mathbb{R}^{n} \ni x=\left(x^{\prime}, y\right), y \in \mathbb{R}$. We may replace the operator $(-\Delta+c)$ in the Definitions $9.1-9.3$ by

$$
-\sum_{i, j=1}^{n-1} \frac{\partial}{\partial x_{i}}\left(a_{i j}\left(x^{\prime}\right) \frac{\partial}{\partial x_{j}}\right)+c\left(x^{\prime}\right)
$$

where the functions $a_{i j}$ and $c$ are bounded and measurable, $c \geq 0$, and the matrix $\left(a_{i j}\right)$ is positive definite, since operator in (10.20) is invariant under the reflection $\sigma_{H}$

2) Applying 1) and following the proofs of this section one generalizes Theorems $10.1-10.3$ as follows. 
Let "*" denote a $(k, n)$-Steiner symmetrization, and let its symmetry plane $\Sigma$ take the form $\{y=0\}$, where $\mathbb{R}^{n} \ni x=\left(x^{\prime}, y\right), y \in \mathbb{R}^{k}$. Then we may replace the operator $(-\Delta+c)$ by

$$
-\sum_{i, j=1}^{n-k} \frac{\partial}{\partial x_{i}}\left(a_{i j}\left(x^{\prime}\right) \frac{\partial}{\partial x_{j}}\right)-\Delta_{y}+c\left(x^{\prime}\right)
$$

where $\Delta_{y}$ denotes the Laplacian with respect to $y$

$$
\Delta_{y}:=\sum_{i=n-k+1}^{n} \frac{\partial^{2}}{\partial x_{i}^{2}},
$$

and the functions $a_{i j}$ and $c$ are as in $\mathbf{1}$ ).

Remark 10.4. An approach via polarization can also be developed for symmetrizations in other measure spaces. For instance it is possible to derive integral inequalities and comparison results for p.d.e.'s for cap symmetrizations on the sphere $S^{n}$ and for symmetrizations in the hyperbolic space $H^{n}$ in a very similar way (compare also [BT], [Bae1], $[\mathrm{Bae2}]$ ).

\section{APPENDiX}

The following convergence property of elliptic boundary value problems in varying domains is well-known (see e.g. $[\mathrm{He}]$ ).

Lemma A. Let $\Omega^{k}, \Omega$ be bounded open sets in $\mathbb{R}^{n}$ such that

$$
\Omega^{k} \subset \Omega^{k+1}, k=1,2, \ldots, \quad \text { and } \bigcup_{k} \Omega^{k}=\Omega .
$$

Let $c \in \mathbb{R}_{0}^{+}$and $f, f^{k} \in L_{+}^{2}(\Omega), k=1,2, \ldots$, such that

$$
f^{k} \longrightarrow f \quad \text { in } L^{2}(\Omega) \text {. }
$$

Further let $u$ and $u^{k}$ be the solutions of the problems $\mathbb{B}_{1}(\Omega, c, f)$ and $\mathbb{B}_{1}\left(\Omega^{k}, c, f^{k}\right)$, respectively, $k=1,2, \ldots$ Then

$$
u^{k} \longrightarrow u \quad \text { in } W_{0}^{1,2}(\Omega)
$$

\section{ACKNOWLEDGMent}

This paper was prepared during a three-month stay at Mathematisches Forschungsinstitut Oberwolfach. We want to express our sincere thanks to the coworkers of the institute for the wonderful atmosphere and working conditions. We thank B. Kawohl, A. Wagner (Cologne), V. Ferone and M. R. Posteraro (Napoli) for some useful conversations. We are also grateful to the referee for some comments which helped us to clarify the exposition and for bringing the article [ALT3] to our attention.

\section{REFERENCES}

[A] H.W. Alt: Lineare Funktionalanalysis. 2nd edn., Springer-Verlag 1992.

[ADLT] A. Alvino, J.I. Diaz, P.-L. Lions, G. Trombetti: Équations elliptiques et symétrization de Steiner. C.R. Acad. Sci. Paris, t.314, Série I (1992), 1015-1020. MR 93f:35047

[ALT1] A. Alvino, P.-L. Lions, G. Trombetti: On optimization problems with prescribed rearrangements. Nonlin. Analysis T.M.A. 13 (1989), 185-220. MR 90c:90236 
[ALT2] A. Alvino, P.-L. Lions, G. Trombetti: Comparison results for elliptic and parabolic equations via symmetrization: A new approach. Differential and Integral Equations 4, (1991), 25-50. MR 91h:35023

[ALT3] A. Alvino, P.-L. Lions, G. Trombetti: Comparison results for elliptic and parabolic equations via Schwarz symmetrization. Ann. Inst. Poincaré, Anal. non lineáire 7(2) (1990), 37-65. MR 91f:35022

[Bae1] A. Baernstein II: Integral means, univalent functions and circular symmetrization. Acta Math. 133 (1974), 139-169. MR 54:5456

[Bae2] A. Baernstein II: A unified approach to symmetrization. in: Partial Differential Equations of Elliptic Type, eds. A. Alvino et al, Symposia matematica 35, Cambridge Univ. Press 1995, 47-91. MR 96e:26019.

[BT] A. Baernstein II, B.A. Taylor: Spherical rearrangements, subharmonic functions, and *-functions in n-space. Duke Math. J. 43 (1976), 245-268. MR 53:5906

[Ban] C. Bandle: Isoperimetric inequalities and applications. Pitman, Boston 1980. MR 81e:35095

[Be] W. Beckner: Sobolev inequalities, the Poisson semigroup and analysis on the sphere $S^{n}$. Proc. Nat. Acad. Sci. U.S.A. 89 (1992), 4816-4819. MR 93d:26018

[BN] H. Berestycki, L. Nirenberg: On the method of moving planes and the sliding method. Bol. Soc. Brasil. Mat. (N.S.) 22 (1991), no.1, 1-37. MR 93a:35048

[BLL] H.J. Brascamp, E.H. Lieb, J.M. Luttinger: A general rearrangement inequality for multiple integrals. J. Funct. Anal. 17 (1974), 227-237. MR 49:10835

[Br] F. Brock: Continuous polarization and Symmetry of Solutions of Variational Problems with Potentials. in: Calculus of variations, applications and computations, Pontà-Mousson 1994, eds. C. Bandle et al., Pitman Research Notes in Math. 326 (1995), 25-35. CMP 97:04

[BS] F. Brock, A. Yu. Solynin: An approach to continuous symmetrization via polarization, in preparation.

[BuZ] Yu.D. Burago, V.A. Zalgaller: Geometric inequalities. Springer- Verlag, Berlin 1988. MR 89b:52020

[CZR] J.A. Crowe, J.A. Zweibel, P.C. Rosenbloom: Rearrangements of functions. J. Funct. Anal. 66 (1986), 432-438. MR 87j:28003

[Da] E.N. Dancer: Some notes on the method of moving planes. Bull. Austr. Math. Soc. 46 (1992), no.3, 425-434. MR 93a:35080

[Di] J.I. Diaz: Nonlinear PDE and free boundaries. Vol.I, Elliptic equations. Pitman Research Notes in Math. 106 (1985). MR 88d:35058

[DS] N. Dunford, J.T. Schwartz: Linear Operators I. Interscience Publishers, New York 1958. MR 22:8302

[Du1] V.N. Dubinin: Transformation of functions and Dirichlet's principle. (in Russian), Math. Zametki 38 (1985), 49-55; English transl. in Math. Notes 38 (1985), 539-542. MR 87j:31005

[Du2] V.N. Dubinin: Transformations of capacitors in space. (in Russian), Dokl. Akad. Nauk SSSR 296 (1988), 18-20, English transl. in Soviet Math. Dokl. 36 (1988), 217-219. MR 89d:31003

[Du3] V.N. Dubinin: Capacities and geometric transformations in n-space. Geom. and Funct. Anal. 3 (1993), 342-369. MR 94f:31008

[Du4] V.N. Dubinin: Symmetrization in geometric theory of functions of complex variables. Uspehi Mat. Nauk 49 (1994), 3-76. MR 96h:30054

[ESh] M. Essén, D.F. Shea: On some questions of uniqueness in the theory of symmetrizations. Ann. Acad. Fenn. Ser.A I, 4 (1978/79), 311-340. MR 81d:30002

[F] A. Friedman: Variational principles and free boundary problems. Wiley- Interscience, N.Y. 1982. [MR 84e:35153

[GNN] B. Gidas, W.-M. Ni, L. Nirenberg: Symmetry and related properties via the maximum principle. Comm. Math. Phys. 68 (1979), 209-243. MR 80h:35043

[GT] D. Gilbarg, N.S. Trudinger: Elliptic partial differential equations of second order. 2nd edn., Springer- Verlag, Berlin 1983. MR 86c:35035

[Ha] H. Hadwiger: Vorlesungen über Inhalt, Oberfläche und Isoperimetrie. Springer- Verlag, Berlin 1957. MR 21:1561 
[He] A. Henrot: Continuity with respect to the domain for the Laplacian: a survey. Control Cybernetics 23(3) (1994), 427-443. MR 95i:35030

[Ka1] B. Kawohl: Rearrangements and Convexity of Level Sets in PDE. Springer lecture notes 1150 (1985). MR 87a:35001

[Ka2] B. Kawohl: On the simple shape of stable equilibria. in: Geometry of Solutions to Partial Differential Equations, ed. G. Talenti, Symposia matematica 30, Academic Press 1989, 73-89. MR 91f:35010

[Kac] J. Kačur: Method of Rothe in Evolution Equations. Teubner-Verlag, Leipzig 1985. MR 87j:35004

[Ke] H.B. Keller: Some positone problems suggested by nonlinear heat generation. in: "Bifurcation Theory and Nonlinear Eigenvalue Problems", ed. J.B. Keller / St. Antman, Benjamin, N.Y. 1969, 217-255.

[Kes1] S. Kesavan: On a comparison theorem via symmetrization. Proc. Roy. Soc. Edinburgh 119 A (1991), 159-167. MR 92h:35057

[Kes2] S. Kesavan: Some remarks on a result of Talenti. Ann. Scuola Norm. Sup. Pisa, Ser. IV, 15 (1991), 453-465. MR 90k:31007

[Kes3] S. Kesavan: Comparison theorems via Schwarz symmetrization - a survey. Symposia mathematica 35 (1994), 185-196. MR 95f:35060

[L] B.E. Levitskii: $k$-symmetrization and extremal rings. (in Russian), Math. Anal. Kuban State Univ. Krasnodar, 1971, 35-40.

[PS] G. Polya, G. Szegö: Isoperimetric inequalities in mathematical physics. Ann. Math. Studies 27 (1952), Princeton Univ. Press. MR 13:270d

[Sa] J. Sarvas: Symmetrization of condensers in n-space. Ann. Acad. Sci. Fenn. Ser. A1, 522 (1972), 1-44. MR 50:606

[Sch] E. Schmidt: Die Brunn-Minkowskische Ungleichung und ihr Spiegelbild sowie die isoperimetrische Eigenschaft der Kugel in der euklidischen und nichteuklidischen Geometrie I,II. Math. Nachrichten 1 (1948), 81-157; 2 (1949), 171-244. [MR 10:471d] [MR 11:5341]

[So1] A.Yu. Solynin: Continuous symmetrization of sets. Zap. Nauchn. Semin. POMI 185 (1990), 125-139. MR 92k:28012

[So2] A. Yu. Solynin: Application of the polarization for proofs of functional inequalities. (in Russian), Russian Academy of Sciences, St. Petersburg, PDMI Preprint 10 (1995).

[St] J. Steiner: Gesammelte Werke. Vol.2, Reimer- Verlag, Berlin 1882.

[Ta1] G. Talenti: Elliptic equations and rearrangements. Ann. Scuola Norm. Pisa Cl. Sci. (4) 3 (1976), 697-718. MR 58:29170

[Ta2] G. Talenti: Best constant in Sobolev inequality. Ann. Mat. Pura Appl., Ser. 4, 110 (1976), 353-372. MR 57:3846

[Ta3] G. Talenti: The standard isoperimetric theorem. in: Handbook of Convex Geometry, Volume A, North-Holland, Amsterdam 1993, 73-124. MR 94h:49065

[We1] A. Weitsman: Spherical symmetrization in the theory of PDE. Comm. PDE 8 (1983), 545-561. MR 85d:35028

[We2] A. Weitsman: Symmetrization and the Poincaré metric. Annals of Math. 124 (1986), 159-169. MR 87h:30051

[Wo] V. Wolontis: Properties of conformal invariants. Amer. Journ. Math., 74 (1952), 587-606. MR 14:36c

Mathematisches Institut, Universität Köln, Weyertal 90, D 50923 Köln, Germany

Current address: Department of Mathematics, University of Missouri-Columbia, Mathematical

Sciences Building, Columbia, Missouri 65211

E-mail address: brock@math.missouri.edu

Russian Academy of Sciences, V.A. Steklov Mathematical Institute, St. Petersburg Branchm, Fontanka 27, 191011 St. Petersburg, Russia

E-mail address: solynin@pdmi.ras.ru 